This article has been accepted for publication in a future issue of this journal, but has not been fully edited. Content may change prior to final publication This work is under IEEE copyright and its published version may be found in http://www.ieeexplore.ieee.org Project acknowledgements: end of document.

\title{
Correlated Multiple Antennas Spectrum Sensing Under Calibration Uncertainty
}

\author{
Zahra Pourgharehkhan $\dagger$, Abbas Taherpour $\dagger$, Member, IEEE, Josep Sala-Alvarez $\ddagger$, Senior Member, IEEE, and \\ Tamer Khattab* ${ }^{*}$ Member, IEEE,
}

\begin{abstract}
We address the problem of spectrum sensing in Cognitive Radios (CRs) when the Secondary User (SU) is equipped with a multiantenna receiver. We consider scenarios with correlation between the received channels at different antennas and unequal per-antenna noise variances to accommodate calibration errors. First, we derive the genie-aided (benchmark) detector with perfect knowledge of the antenna correlation coefficients, Primary User (PU) signal power and noise covariance matrix, as well as its asymptotic performance. Then, we consider the sensing problem in which the $\mathrm{SU}$ is non-cognizant of the perantenna noise variances, the $\mathrm{PU}$ signal power and the correlation of channel gains, with a specific treatment of the two-antenna case. For a general multiantenna receiver, we propose to combine the derived test statistics among all antenna pairs. The related optimization problem to obtain the optimum combination weights is analyzed, which requires the analytical performance characterization of the constituent two-antenna detector. Thus, we compute the exact performance of the proposed detector in a special case (a particular case of the Hadamard Ratio test) in terms of its detection and false alarm probabilities. Performance analyses are verified with simulations, showing that the proposed detector outperforms several recently-proposed multiantenna detectors for CR in the scenarios considered.
\end{abstract}

\section{INTRODUCTION}

The Radio Spectrum has become a very demanded and valuable resource due to the technological developments in wireless communication. Meanwhile, significantly under-utilized licensed frequency bands as a function of time or location have been observed. CR allows for opportunistic usage of these spectrum holes by unlicensed users (SUs) when the licensed user (PU) is absent. Therefore, spectrum sensing to detect the absence or presence of the PU's [1] constitutes the first task of a cognitive receiver. Reliable and accurate spectrum sensing without harmful interference to PUs has been treated by many authors [2]. Energy Detection (ED) is a common approach because of its simplicity: in white noise, it requires no knowledge about the PU signal [3], [4]. Unfortunately, its performance degrades under uncertainty in the noise variance(s) [5]. The use of multiple antennas has been proposed to overcome noise uncertainty and limited single-receiver sensing capabilities due to channel fading [1], [2], [6]-[8]. Some proposed multiple antenna schemes based on the ED require exact knowledge about noise variances of antennas and thus, remain sensitive to the noise uncertainty [4], [9]. Due to its parameter estimation capability, the Generalized Likelihood Ratio Test (GLRT) is a popular approach to combatting noise uncertainty [1], [2], [6], [8], [10]. If, in addition to noise uncertainty, we contemplate random spatially correlated perantenna channel gains as evidenced for some scenarios, the existing multiantenna spectrum sensing techniques can be classified under four categories,
1) correlation-unaware schemes for calibrated arrays: this constitutes the commonest study case. For instance, the John's detector under multiple PU's [11] (optimum in the low-SNR regime) or proposed detectors. In practice, the calibration errors are unavoidable such that noise variances of antennas can become different. Thus, although these detectors are robust to noise variance uncertainty, none of them is efficient in the uncalibrated case, which may degrade their performance.

2) correlation-unaware schemes for uncalibrated arrays: for instance, GLRT eigenvalue-based detectors for multiantenna spectrum sensing of a PU signal with spatial rank larger than one have been derived [8] for unequal per-antenna noise variances.

However, signals from far PUs arrive at the SU base station within a small beamwidth, which results in a high spatial correlation between the antenna channel gains. As the beamwidth of the received signal decreases, antenna correlations get stronger [12]. As an example, we may refer to IEEE 802.22 Wireless Regional Area Network (WRAN) systems, a standard for using white spaces in the TV frequency spectrum [13], [14] in which PUs are so far from SUs. This fact motivates the following two categories.

3) correlation-aware schemes for calibrated arrays: for instance, [4] evaluates the performance of the ED with correlated antennas and so, is dependent on the noise variance. The detectors in [15] require complete knowledge about the covariance matrix of the PU signal while one of them suffers noise mismatch. Finally, [16] has considered an SU receiver with a linear equispaced antenna array when channel gains undergo block fading and where the proposed detectors, based on interantenna path correlation, are blind up to knowledge of the maximum beamwidth of the received signals. As beamwidth is not exactly known in wireless environments, some performance degradation is incurred in practice.

4) correlation-aware schemes for uncalibrated arrays: this category is seldom treated. For instance, see [17].

As exposed in the fourth category, this paper address the problem of multiantenna spectrum sensing considering the correlation between the received channels at different uncalibrated antennas in the presence of Additive White Gaussian Noise (AWGN). Section II describes the system model and basic assumptions. In Section III, we derive the optimum genieaided detector operating on perfect knowledge of the antenna correlation coefficients, PU signal power and antenna noise 
variances: a necessary benchmark for comparison with more practical detectors for which these parameters are unknown. We evaluate the performance of the optimum and proposed detectors in terms of detection and false alarm probabilities. In Section IV, we consider a fully blind detector for which no knowledge of the PU signal power and the antenna noise variances, nor of the correlation between channel gains, is available. In this case, we show that the blind detector is a special case of the Hadamard ratio test in [17]. For solving the sensing problem, we first consider that the SU is fitted with only two antennas. Then, for the general case, we propose to combine weighted test statistics among all antenna pairs. In a special case, the proposed detector turns out to be equal to the ad hoc detector $\|\widehat{\mathbf{C}}\|_{F}^{2}$ in [17] (Frobenius norm of the coherence matrix). To determine the optimum weighting coefficients, we need to derive the exact performance of the two-antenna detector in terms of false alarm and detection probabilities. The asymptotical results are also analytically provided in this case. Simulation results in Section V verify that the proposed detector outperforms several recentlyproposed multiantenna detectors for CR. Finally conclusions are drawn in Section VI. Section VII contains the appendices with the more detailed mathematical derivations.

Notation: Boldface lower- and upper-case letters are used respectively to denote column vectors and matrices. We let $\mathbf{X}_{(m n)}$ denote the $(m n)^{\text {th }}$ element of matrix $\mathbf{X}$. The $m^{\text {th }}$ column of matrix $\mathbf{X}$ is denoted $\mathbf{x}^{(m)}$, while the $\ell^{\text {th }}$ element of vector $\mathbf{x}$ is denoted $\mathbf{x}_{\ell}$. We use $\operatorname{tr}(\mathbf{X})$ and $\operatorname{det}(\mathbf{X})$ for the trace and determinant of matrix $\mathbf{X}$, respectively. $\mathbf{X}^{T}$ and $\mathbf{X}^{H}$ stand for the transpose and Hermitian of matrix $\mathbf{X}$, respectively. Additionally, $\operatorname{diag}\{\mathbf{x}\}$ constitutes a square matrix with the elements of $\mathbf{x}$ along its main diagonal and zeros elsewhere. Given a complex number $z,|z|, \mathfrak{R}\{z\}$ and $\mathfrak{I}\{z\}$ denote the magnitude, real and imaginary parts of $z$, respectively. In addition, Kroneckers product and the identity matrix of size $L$ are represented as $\otimes$ and $\mathbf{I}_{L}$, respectively. A vector with all elements equal to 1 is denoted $\mathbf{1}$. We define the expectation and variance of a random variable $\mathbf{x}$ as $\mathbb{E}[\mathbf{x}]$ and $\operatorname{Var}(\mathbf{x})$, respectively. $\operatorname{Cov}(x, y)$ is employed for the covariance between random variables $x$ and $y$. We use $\mathcal{C N}(\mathbf{0}, \boldsymbol{\Sigma})$ to represent the complex Gaussian distribution with zero mean and covariance matrix $\boldsymbol{\Sigma}$. Finally, $N\left(\mu, \sigma^{2}\right)$ is used to represent a real Gaussian random variable with mean $\mu$ and variance $\sigma^{2}$.

\section{BASIC ASSUMPTION AND SyStem Model}

We consider an $M$-antenna SU receiver performing spectrum sensing. Over the observation period, $L$ consecutive complex samples at the Nyquist rate $f_{s}$ are captured at the output of each antenna's radio-frequency front-end, after down-converting to the baseband in-phase and quadrature components of the received passband signal. We let hypotheses $\mathcal{H}_{1}$ and $\mathcal{H}_{0}$ denote, respectively, the presence and absence of the PU signal. The equivalent binary hypothesis testing problem at the $m^{\text {th }}$ antenna and $\ell^{\text {th }}$ time instant is then formulated as,

$$
\mathbf{y}_{\ell}^{(m)}=\left\{\begin{array}{ccc}
\mathbf{n}_{\ell}^{(m)} & , & \mathcal{H}_{0} \\
\mathbf{h}_{\ell}^{(m)} \mathbf{s}_{\ell}+\mathbf{n}_{\ell}^{(m)} & , & \mathcal{H}_{1}
\end{array}, \quad m=1,2, \ldots, M,\right.
$$

with $\mathbf{y}^{(m)} \in \mathbb{C}^{L}$ the vector of the received complex baseband signal at the $m^{\text {th }}$ antenna and $\mathbf{Y}=\left[\mathbf{y}^{(1)}, \cdots, \mathbf{y}^{(M)}\right] \in \mathbb{C}^{L \times M}$ the corresponding time $\times$ antenna data matrix. We take vector $\mathbf{s}$ to be a zero-mean i.i.d modulated signal sequence of power $\mathcal{E}_{s}$ modeling the PU's transmission. We let $\mathbf{n}^{(m)} \in \mathbb{C}^{L}$ denote the $m^{\text {th }}$ antenna zero-mean circular complex additive white Gaussian noise vector and $\mathbf{N}$ its corresponding time $x$ antenna data matrix. The noise spatial covariance matrix is $\boldsymbol{\Sigma}_{N} \triangleq \frac{1}{L} \mathbb{E}\left\{\mathbf{N}^{H} \mathbf{N}\right\}=\operatorname{diag}\left\{\sigma_{1}^{2}, \cdots, \sigma_{M}^{2}\right\}$. We model the channel gains vector $\mathbf{h}^{(m)} \in \mathbb{C}^{L}$ between the PU and $m^{\text {th }}$ antenna as a spatially correlated zero-mean circular complex Gaussian random process. This channel model, successfully applied in other works [2] [18], proves helpful in that: (i) admitting channel variability within the observation window, it does not settle for any specific channel coherence time; (ii) for a constant modulus signal $\mathbf{s}$ with i.i.d. samples and uniformly distributed phase, the statistical distribution under $\mathcal{H}_{1}$ is still Gaussian, which is amenable to theoretical derivations; (iii) the resulting detectors turn out to be expressed in terms of sample moments of the received signal - which, together with performance analysis, are validated in the simulations section over more practical scenarios and other signal models where $\mathbf{s}$ is not necessarily constant modulus i.i.d. - . A more extended discussion of these aspects of the signal model may be found in [18]. Considering these assumptions, the data matrix $\mathbf{Y}$ is modeled with independent zero-mean rows, so that, for $1 \leq i \leq L$, we may rewrite (1) as,

$$
\left\{\begin{array}{lll}
\mathcal{H}_{0}: & \operatorname{row}_{i}[\mathbf{Y}] \sim \mathcal{C N}\left(\mathbf{0}, \boldsymbol{\Sigma}_{0}\right), & \text { PU is absent } \\
\mathcal{H}_{1}: & \operatorname{row}_{i}[\mathbf{Y}] \sim \mathcal{C N}\left(\mathbf{0}, \boldsymbol{\Sigma}_{1}\right), & \text { PU is present. }
\end{array}\right.
$$

where $\boldsymbol{\Sigma}_{\eta}$ denotes the covariance matrix of the $i^{\text {th }}$ row of $\mathbf{Y}$ under hypothesis $\mathcal{H}_{\eta}$, with,

$$
\boldsymbol{\Sigma}_{0}=\boldsymbol{\Sigma}_{N} \quad, \quad \boldsymbol{\Sigma}_{1}=\mathcal{E}_{s} \boldsymbol{\Sigma}_{H}+\boldsymbol{\Sigma}_{N}
$$

where $\boldsymbol{\Sigma}_{H}$ is the covariance matrix of $\mathbf{h} \triangleq$ $\left[\mathbf{h}_{\ell}^{(1)}, \mathbf{h}_{\ell}^{(2)}, \cdots, \mathbf{h}_{\ell}^{(M)}\right]^{T}$ for $\ell=1, \cdots, L$. Some works have established a model for $\mathbf{C}$ from the physical parameters of the scenario, for one- and two-dimensional antenna arrays [19] receiving a far source with angles of arrival of each path distributed within a beamwidth $[\phi-\Delta, \phi+\Delta]$ [20] [16] (related with the correlation of the antenna gains). Those models, also contemplated in [18], reduce to Clark's model [21] for the parameter settings $\phi=0$ and $\Delta=\pi$. The expressions for $\mathbf{C}$ in [18] (Eqs. 6,7,8) have been used in the simulations section as a validation scenario.

\section{OPTIMUM DETECTOR}

According to the model in (1), the presence of the PU is revealed by the correlation between the signals received at each antenna. In this section, we derive the optimal NeymanPearson (NP) detector corresponding to the case in which the SU has complete knowledge of the covariance matrices $\boldsymbol{\Sigma}_{0}$ 
and $\boldsymbol{\Sigma}_{\mathbf{1}}$. From (2), the Probability Density Function (PDF) of the observations matrix $\mathbf{Y}$ under hypothesis $\mathcal{H}_{\eta}$ is as follows,

$$
f\left(\mathbf{Y} \mid \mathcal{H}_{\eta}, \boldsymbol{\Sigma}_{\eta}\right)=\frac{\exp \left\{-\operatorname{tr}\left(\mathbf{V} \boldsymbol{\Sigma}_{\eta}^{-1}\right)\right\}}{\pi^{2 L} \operatorname{det}\left(\boldsymbol{\Sigma}_{\eta}\right)^{L}} .
$$

where $\mathbf{V} \triangleq \mathbf{Y}^{H} \mathbf{Y}$ is scatter matrix of $\mathbf{Y}$. Thus, the NP detector is formed as,

$$
\operatorname{LR}=\frac{f\left(\mathbf{Y} \mid \mathcal{H}_{1}, \boldsymbol{\Sigma}_{1}\right)}{f\left(\mathbf{Y} \mid \mathcal{H}_{0}, \boldsymbol{\Sigma}_{0}\right)}
$$

Taking the logarithm in (5) and defining $\mathcal{L}_{\eta}(\mathbf{Y}) \triangleq$ $\ln f\left(\mathbf{Y} \mid \mathcal{H}_{\eta}, \boldsymbol{\Sigma}_{\eta}\right)$, we have,

$$
\begin{aligned}
\mathrm{LLR} & =\ln \frac{f\left(\mathbf{Y} \mid \mathcal{H}_{1}, \boldsymbol{\Sigma}_{1}\right)}{f\left(\mathbf{Y} \mid \mathcal{H}_{0}, \boldsymbol{\Sigma}_{0}\right)}=\mathcal{L}_{1}(\mathbf{Y})-\mathcal{L}_{0}(\mathbf{Y}) \\
& =L \operatorname{tr}\left(\mathbf{S}\left(\boldsymbol{\Sigma}_{0}^{-1}-\boldsymbol{\Sigma}_{1}^{-1}\right)\right)+L \ln \frac{\operatorname{det}\left(\boldsymbol{\Sigma}_{0}\right)}{\operatorname{det}\left(\boldsymbol{\Sigma}_{1}\right)}
\end{aligned}
$$

where $\mathbf{S}=\frac{1}{L} \mathbf{V}$ which is known as sample covariance matrix. The derived decision statistic should be compared with a threshold. By absorbing the constant terms into the decision threshold, the resulting detector will be,

$$
T_{\text {opt }}=\operatorname{tr}(\mathbf{S} \Sigma) \underset{\mathcal{H}_{0}}{\stackrel{\mathcal{H}_{1}}{\gtrless}} \lambda .
$$

where $\boldsymbol{\Sigma} \triangleq \boldsymbol{\Sigma}_{0}^{-1}-\boldsymbol{\Sigma}_{1}^{-1}$ and $\lambda$ is decision threshold. In the following section, we obtain an approximate expression for the asymptotic $(L \rightarrow \infty)$ performance of optimum detector.

\section{A. Performance of the Optimum Detector}

The approximate performance of the optimum detector is evaluated in terms of its detection and false-alarm probabilities, $P_{\mathrm{d}}$ and $P_{\mathrm{fa}}$, respectively. To that purpose, we need to compute the distribution of the decision statistics under hypotheses $\mathcal{H}_{0}$ and $\mathcal{H}_{1}$, which we will approximate by a Gaussian random variable (sample moments of random data with finite fourth-order moments are asymptotically Gaussian distributed [22]). To the best of our knowledge, a closed form solution is not known. The sample covariance matrix $\mathbf{S}$ is complex Wishart distributed with $L$ degrees of freedom and parameter matrices $\boldsymbol{\Sigma}_{0}$ and $\boldsymbol{\Sigma}_{1}$ under $\mathcal{H}_{0}$ and $\mathcal{H}_{1}$, respectively. As a Gaussian distribution is completely described by its mean and variance, we only need to compute the two first moments of the decision statistic. Using the moments provided in [23], the mean and standard deviation of $T_{\text {opt }}$ under $\mathcal{H}_{\eta}$, denoted respectively as $m_{\eta}^{\mathrm{opt}}$ and $\nu_{\eta}^{\mathrm{opt}}$, are found as,

$$
\begin{aligned}
m_{0}^{\mathrm{opt}}=M-\operatorname{tr}\left(\boldsymbol{\Sigma}_{N}\left(\boldsymbol{\Sigma}_{1}\right)^{-1}\right) \quad, \quad m_{1}^{\mathrm{opt}}=\operatorname{tr}\left(\boldsymbol{\Sigma}_{1}\left(\boldsymbol{\Sigma}_{N}\right)^{-1}\right)-M \\
\nu_{0}^{\mathrm{opt}}=\sqrt{\frac{1}{L} \operatorname{tr}\left(\left(\boldsymbol{\Sigma} \boldsymbol{\Sigma}_{N}\right)^{2}\right)} \quad, \quad \nu_{1}^{\mathrm{opt}}=\sqrt{\frac{1}{L} \operatorname{tr}\left(\left(\boldsymbol{\Sigma} \boldsymbol{\Sigma}_{1}\right)^{2}\right)}
\end{aligned}
$$

Thus, the false alarm and detection probability are respectively calculated as

$$
P_{\mathrm{fa}}^{\mathrm{opt}}=Q\left(\frac{\lambda-m_{0}^{\mathrm{opt}}}{\nu_{0}^{\mathrm{opt}}}\right)
$$

$$
P_{\mathrm{d}}^{\mathrm{opt}}=Q\left(\frac{\lambda-m_{1}^{\mathrm{opt}}}{\nu_{1}^{\mathrm{opt}}}\right)=Q\left(\frac{\nu_{0}^{\mathrm{opt}} Q^{-1}\left(P_{\mathrm{fa}}\right)+m_{0}^{\mathrm{opt}}-m_{1}^{\mathrm{opt}}}{\nu_{1}^{\mathrm{opt}}}\right)
$$

where $Q(\cdot)$ is the tail probability of the standard normal distribution and $Q^{-1}(\cdot)$ its inverse.

\section{Proposed Detector}

We consider the optimum NP detector only for benchmark purposes. In practice, the SU does not possess any knowledge of the matrices $\boldsymbol{\Sigma}_{0}$ and $\boldsymbol{\Sigma}_{1}$, in which case, they should be estimated. Known solutions, for instance GLRT-based detectors [1], can be used in these situations. In addition it is also of interest to derive the analytical performance of the known and proposed detectors.

First, we consider a special case: a detector for the SU that only uses two antenna elements (the $m^{\text {th }}$ and $n^{\text {th }}$ antennas). Under the GLRT framework, we can use the Maximum Likelihood Estimation (MLE) of the unknown parameters and then form the test statistic as,

$$
\mathrm{LR}=\frac{f\left(\mathbf{Y} \mid \mathcal{H}_{1}, \widehat{\boldsymbol{\Sigma}}_{1}\right)}{f\left(\mathbf{Y} \mid \mathcal{H}_{0}, \widehat{\boldsymbol{\Sigma}}_{0}\right)}
$$

where $\widehat{\boldsymbol{\Sigma}}_{\eta}=\underset{\boldsymbol{\Sigma}_{\eta}}{\arg \max } f\left(\mathbf{Y} \mid \mathcal{H}_{\eta}, \boldsymbol{\Sigma}_{\eta}\right)$. From [17], the corresponding two-antenna blind detector becomes,

$$
T_{\text {SPCL }}=\left|\hat{R}_{m, n}\right|^{2} \stackrel{\mathcal{H}_{1}}{\underset{\mathcal{H}_{0}}{\gtrless}} \lambda \text {. }
$$

with $\hat{R}_{m, n} \triangleq \frac{\mathbf{V}_{(m n)}}{\sqrt{\mathbf{V}_{(m m)} \mathbf{V}_{(n n)}}}$. In fact, we are estimating the available correlation between two antennas, a special case of Hadamard ratio detector [17] defined as $T_{\mathrm{H}}=\frac{\prod_{i=1}^{M} \mathbf{V}_{(i i)}}{\operatorname{det}(\mathbf{V})}$. We may incorporate more antennas to achieve an adequate diversity gain. To this end, we propose to combine the weighted estimated correlations between all the possible antennas pairs as,

$$
T_{\text {Prop }}=\mathbf{w}^{T} \hat{\mathbf{r}} \underset{\mathcal{H}_{0}}{\stackrel{\mathcal{H}_{1}}{\gtrless}} \tau .
$$

where $\mathbf{w}=\left[\begin{array}{llll}\mathbf{w}_{1}, & \mathbf{w}_{2}, & \cdots, & \mathbf{w}_{N}\end{array}\right]^{T} \in R^{N}$ is weighting vector in which $N=\frac{M(M-1)}{2}$. In addition, $\hat{\mathbf{r}}=$ $\left[\left|\hat{R}_{1,2}\right|^{2},\left|\hat{R}_{1,3}\right|^{2}, \cdots,\left|\hat{R}_{M-1, M}\right|^{2}\right]^{T} \in R^{N}$ is estimated correlation vector of all antenna pairs. In fact, proposed detector in special case of equal gains is exactly the ad hoc detector $\|\widehat{\mathbf{C}}\|_{F}$ proposed in [17] (which was shown to closely approximate the asymptotic low-SNR GLRT: the Hadamard ratio detector). Since, $\|\widehat{\mathbf{C}}\|_{F}^{2}$ is twice sum of the squares of the correlation estimation of all antenna pairs plus a constant value. Our goal is to improve on the performance of test $\|\widehat{\mathbf{C}}\|_{F}$ by computing a gain vector $\mathbf{w}^{*}$ as a solution of an appropriate optimization problem. To this end, it is required that the detection and falsealarm probabilities be available in analytical form. To the best of our knowledge, such analytical results are not available yet in the literature. We proceed now to obtain the required exact and asymptotic performance expressions.

Lemma 1. The special detector $T_{\mathrm{SPCL}}$ under $\mathcal{H}_{0}$ for any arbitrary pair of antennas is distributed as $\operatorname{Beta}(1, L-1)$, with an exact false-alarm probability given by,

$$
P_{\mathrm{fa}}^{\mathrm{SPCL}}=P\left[\left|\hat{R}_{m, n}\right|^{2}>\lambda \mid \mathcal{H}_{0}\right]=I_{1-\lambda}(L-1,1)
$$


with Beta $(\alpha, \beta)$ the Beta distribution with shape parameters $\alpha$ and $\beta$ and $I_{x}(a, b)=\frac{1}{B(a, b)} \int_{0}^{x} t^{a-1}(1-t)^{b-1} \mathrm{~d} t$ the regularized incomplete beta function. $T_{\mathrm{SPCL}}$ 's exact detection probability is given by,

$$
\begin{aligned}
& P_{\mathrm{d}}^{\mathrm{SPCL}}= \\
& P\left[\left|\hat{R}_{m, n}\right|^{2}>\lambda \mid \mathcal{H}_{1}\right]=1-\sum_{k=0}^{\infty} p_{k} I_{\lambda}(1+k, L-1) \\
& =\sum_{k=0}^{\infty} p_{k} I_{1-\lambda}(L-1,1+k)
\end{aligned}
$$

where

$$
p_{k}=\frac{L^{(k)}}{k !}\left(1-\left|R_{m, n}\right|^{2}\right)^{L}\left|R_{m, n}\right|^{2 k}
$$

in which $x^{(n)}=x(x+1)(x+2) \cdots(x+n-1)$ is the rising factorial of $x$ (Pochhammer's function) and $\left|R_{m, n}\right|^{2}=$ $\frac{\mid \boldsymbol{\Sigma}_{\left.1_{(m n)}\right|^{2}}}{\boldsymbol{\Sigma}_{1_{(m m)} \boldsymbol{\Sigma}_{1(n n)}}}$ the actual correlation between the $m^{\text {th }}$ and $n^{\text {th }}$
antennas.

\section{Proof: See appendix VII-A}

For two antennas, Lemma 1 provides the exact probabilities of a special case of Hadamard ratio detector for two antennas. The complexity of those expressions lets us surmise that computing the exact probabilities for $T_{\text {Prop }}$ in (13) will lead to an intractable problem if we intend to optimize the detector's performance over $\mathbf{w}$. Thus, we adopt a relaxed approach and employ instead the approximate asymptotic distributions in Lemma 2 to define a suitable and mathematically tractable optimization criterion.

Lemma 2. If the number of sensing samples $L$ is large enough, and letting the symbol $\stackrel{\text { ap. }}{\sim}$ denote 'approximately distributed as', we have, under hypotheses $\mathcal{H}_{0}$ and $\mathcal{H}_{1}$,

$$
\begin{array}{lll}
T_{\text {Prop }} \mid \mathcal{H}_{0} & \stackrel{\text { ap. }}{\sim} & N\left(\frac{1}{L} \mathbf{w}^{T} \mathbf{1}, \frac{1}{L^{2}}\|\mathbf{w}\|^{2}\right) \\
T_{\text {Prop }} \mid \mathcal{H}_{1} & \stackrel{\sim}{\text { ap. }} & N\left(\mathbf{w}^{T} \boldsymbol{\mu}_{1}, \mathbf{w}^{T} \mathbf{\Theta} \mathbf{w}\right)
\end{array}
$$

with $\boldsymbol{\mu}_{1}=\left[\mu_{1}(1,2), \mu_{1}(1,3), \cdots, \mu_{1}(M-1, M)\right]^{T}$, in which $\mu_{1}(m, n)=\mathbb{E}\left[\left|\hat{R}_{m, n}\right|^{2} \mid \mathcal{H}_{1}\right]$ is available in (44) and in (45) in its exact and asymptotic forms, respectively. In addition, $\Theta$ is the covariance matrix of $\mathbf{r}$, where the asymptotic form of each component is as specified in (58).

Remark: $T_{\text {Prop }}$ in (13) is a finite addition of $N=\frac{M(M-1)}{2}$ random variables. The goodness of the approximate distributions (17) and (18) is respectively examined in Fig. 4 and Fig. 5 for several value of $L$ by comparing the Cumulative Distribution Function (CDF) of the test statistic with its fitting Gaussian distribution.

\section{Proof: Refer to VII-B}

We are already in a position to define an optimization problem for the weight vector $\mathbf{w}$ to be used in the test statistic (13): the optimum weights $\mathbf{w}^{*}$ will be those maximizing the following (asymptotic) detection probability function, subject to the specified chief constraint on the (asymptotic) false-alarm function and to the necessary parameter-related constraints,

$$
\begin{aligned}
\max _{\mathbf{w}, \eta}: & P_{\mathrm{d}}^{\mathrm{prob}}(\mathbf{w}, \eta) \\
\text { s.t. }: & P_{\mathrm{fa}}^{\mathrm{prop}} \leq \beta \quad, \quad \beta<1 / 2 \\
& \mathbf{w}, \eta>0
\end{aligned}
$$

where $\beta$ is a predefined constraint on the false alarm probability to guarantee more than $50 \%$ opportunistic utilization of spectrum holes.

Lemma 3. The optimization problem (19) can be re-expressed as

$$
\min _{\mathbf{w}>0}: \frac{Q^{-1}(\beta)\|\mathbf{w}\| / L-\mathbf{q}^{T} \mathbf{w}}{\sqrt{\mathbf{w}^{T} \boldsymbol{\Theta} \mathbf{w}}}
$$

where $\mathbf{q} \triangleq \boldsymbol{\mu}_{1}-1 / L \mathbf{1}$. We note that $Q^{-1}(\beta)>0$ and that the solution to problem (20) is invariant to scalings of $\mathbf{w}$. Thus, the optimization problem can be replaced by

$$
\begin{array}{ll}
\min _{\mathbf{z}>0}: & Q^{-1}(\beta)\|\mathbf{z}\| / L-\mathbf{q}^{T} \mathbf{z} \\
\text { s.t. : } & \mathbf{z}^{T} \boldsymbol{\Theta} \mathbf{z} \leq 1
\end{array}
$$

which is a simple convex optimization problem that can be easily solved, with optimum weights,

$$
\mathbf{w}^{*}=\mathbf{z}^{*} \sqrt{\mathbf{z}^{*^{T}} \boldsymbol{\Theta} \mathbf{z}^{*}}
$$

Proof: Refer to [24].

Since we are assuming the detector to be blind, the exact values of $\mathbf{q}$ and $\Theta$ are not available to solve the optimization problem. However, we can use their estimates. The exact values of $\mathbf{q}$ and $\Theta$ are functions of the correlation values between the antennas pairs $\left|R_{m, n}\right|^{2}$ for $m=1, \cdots, M-1$ and $n=m+1, \cdots, M$ and thus, we can easily replace them with their estimates $\left|\hat{R}_{m, n}\right|^{2}$.

\section{Simulation}

In this section, some simulation results are provided to evaluate the performance of the proposed detector and verify analytical results. In our simulation, the average received SNR at SU has been defined as

$$
S N R \triangleq \frac{\mathcal{E}_{s} \operatorname{tr}(\mathbf{C})}{\operatorname{tr}\left(\boldsymbol{\Sigma}_{N}\right)}
$$

We have assumed that the SU is equipped with a linear equispaced antenna array with inter-element spacing $D$. As discussed, the correlation of channel coefficients increases with decreases in $D$. As realized, our proposed spectrum sensing approach depends on correlation between different antennas and clearly, decreases in $D$ would improve the performance of the proposed detector. However, we should note that inter-element spacing cannot be selected arbitrary small in practice. Because, small inter-element spacing yields mutual coupling between the antennas which results in an undesirable correlation of the received signals among the antennas for spacings below one wavelength [25], [26]. Although the signal coupling does not affect the correlation between channels [27], the noise correlation caused by the signal coupling degrades the performance of proposed scheme. Therefore, an 


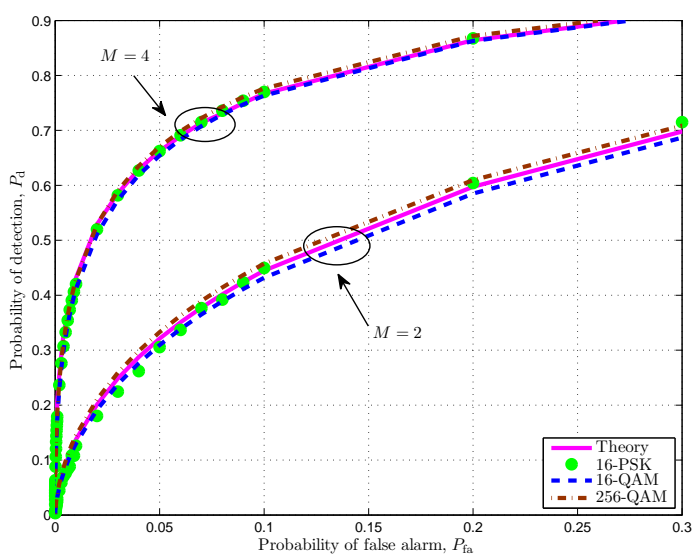

Fig. 1. ROC curves of proposed detector based on some modulation, when $\mathbf{w}=\mathbf{1}$ for $S N R=-6(d B), L=50$ and $\Delta=\pi / 8$.

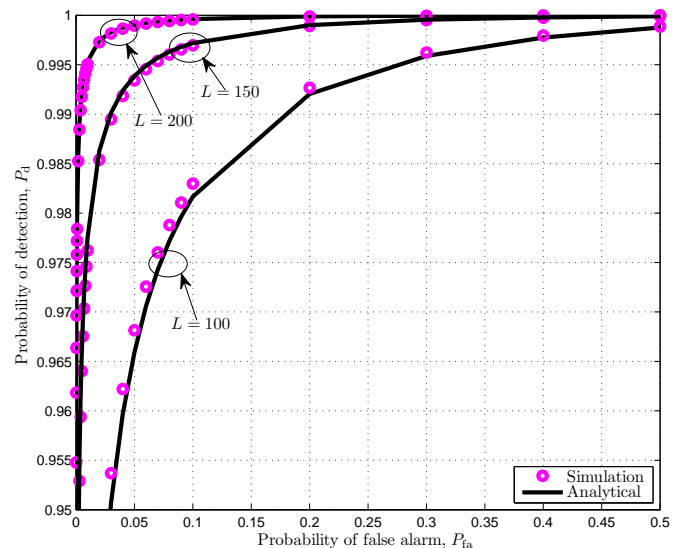

Fig. 2. Comparison between simulation and analytical performance of the optimal detector when $M=4$, for $S N R=-10(d B)$ and $\Delta=\pi / 8$.

appropriate value for antenna spacing should be chosen so that noise samples remain independent of each other among antennas. On the other hand, inter-element spacing must not be so large that the correlation of the channel gains becomes negligible. Thus, we should consider some values of $D$ so that both conditions become satisfied. As an appropriate choice, we consider values for $D$ satisfying $D / \lambda_{c}=0.39$ [16], [27].

It has been assumed that $\phi=0$ and the modulation scheme for transmitted signals has been considered 16-QAM.

\section{A. Verification of Analytical Results}

In our system model, we assumed that the received signal samples are Gaussian under both hypotheses. When PU is absent, this assumption is almost always true due to thermal noise. As mentioned, this assumption is true for modulation schemes which all the signals in the constellation have equal magnitude, for instance M-PSK. We demonstrate that this assumption is acceptable for other modulation schemes as well. We have plotted the receiver operating characteristics (ROC) curves in Fig. 1 when transmitted signal is 16-PSK, and 16-QAM and 256-QAM and compared them with Gaussian

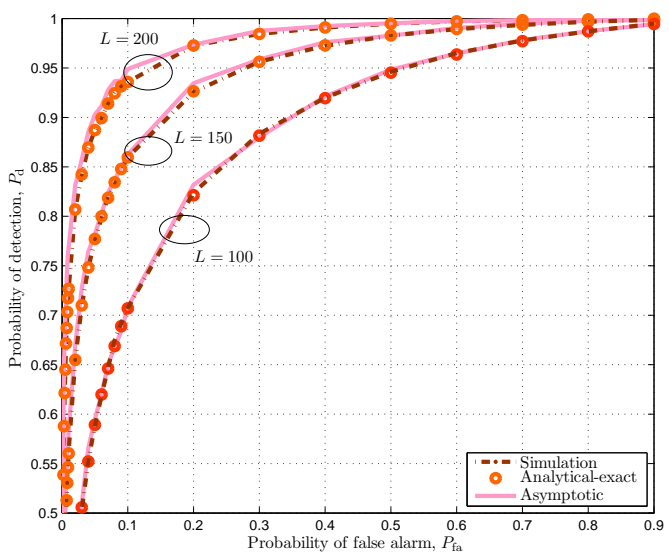

Fig. 3. Comparison between simulation and analytical performance of the proposed detector in special case $(M=2)$, for $S N R=-6(d B)$ and $\Delta=$ $\pi / 8$.

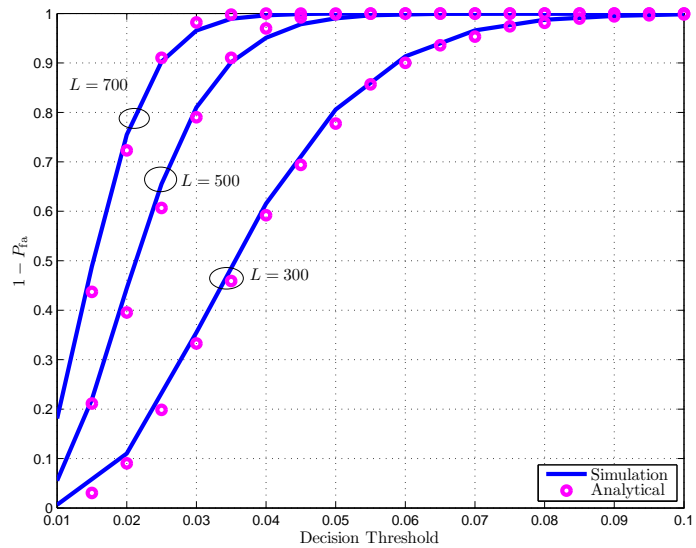

Fig. 4. Comparison between simulation and analytical CDF of the proposed decision statistic under $\mathcal{H}_{0}$ when $M=4$ and $\mathbf{w}=$ $[1.6394,2.0275,2.0734,2.1830,1.2226,2.0924]^{T}$.

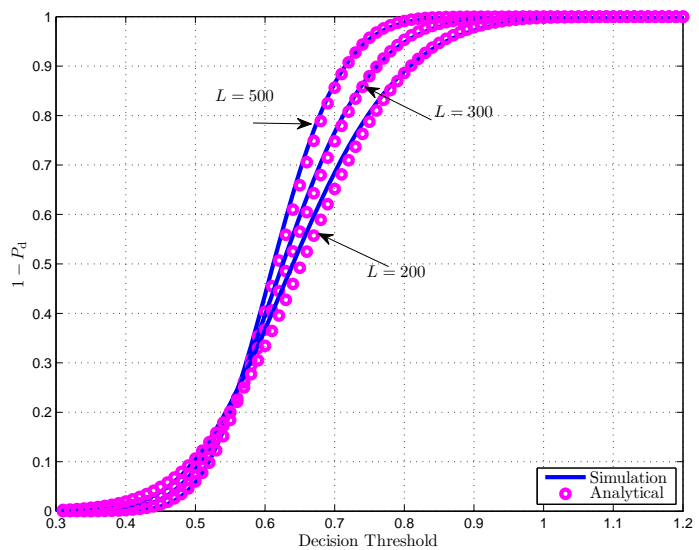

Fig. 5. Comparison between simulation and analytical CDF of the proposed decision statistic under $\mathcal{H}_{1}$ when $M=4$, for $S N R=-5(d B), \Delta=\pi / 8$ and $\mathbf{w}=[1.6394,2.0275,2.0734,2.1830,1.2226,2.0924]^{T}$.

assumption. It has been assumed that $S N R=-6(d B)$, $L=50$ and $\Delta=\pi / 8$ when $\mathbf{w}=1$. As observed, there 


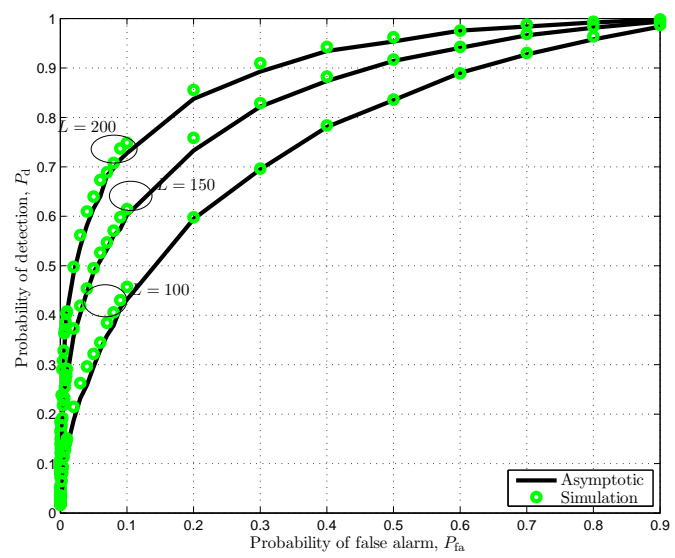

Fig. 6. Comparison between simulation and analytical performance of $\|\widehat{\mathbf{C}}\|_{F}^{2}$, for $S N R=-10(d B), M=4$ and $\Delta=\pi / 8$.

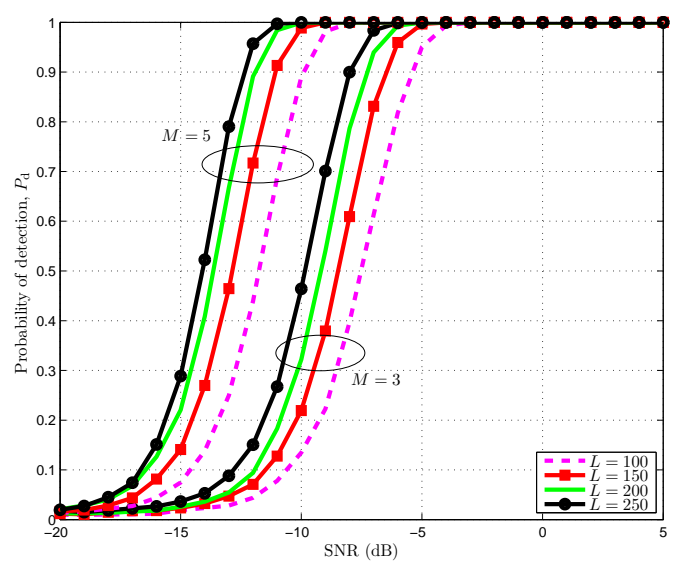

Fig. 7. Detection probability of proposed detector versus SNR, for $P_{\mathrm{fa}}=0.01$ and $\Delta=\pi / 8$.

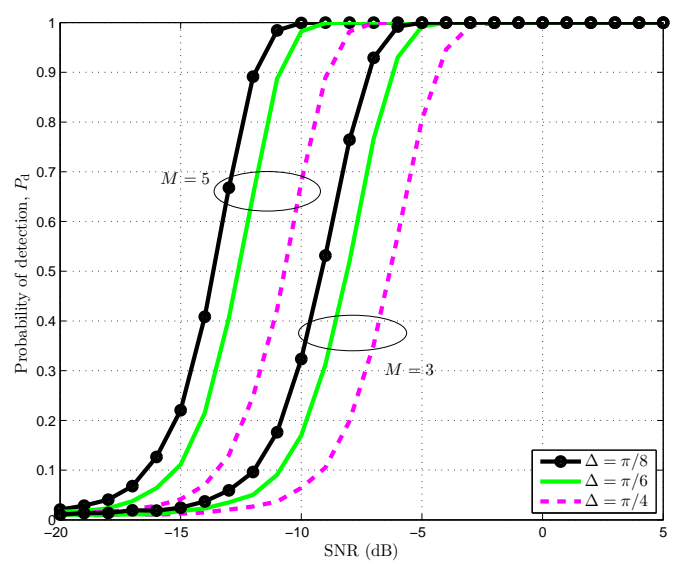

Fig. 8. Detection probability of proposed detector versus SNR, for $P_{\mathrm{fa}}=0.01$ and $L=200$.

is no significant difference between the numerical and analytical results. Fig. 2 depicts the ROC curves of analyticalbased performance of optimal detector and simulation-based performance. In this figure, it is assumed that $M=4$,

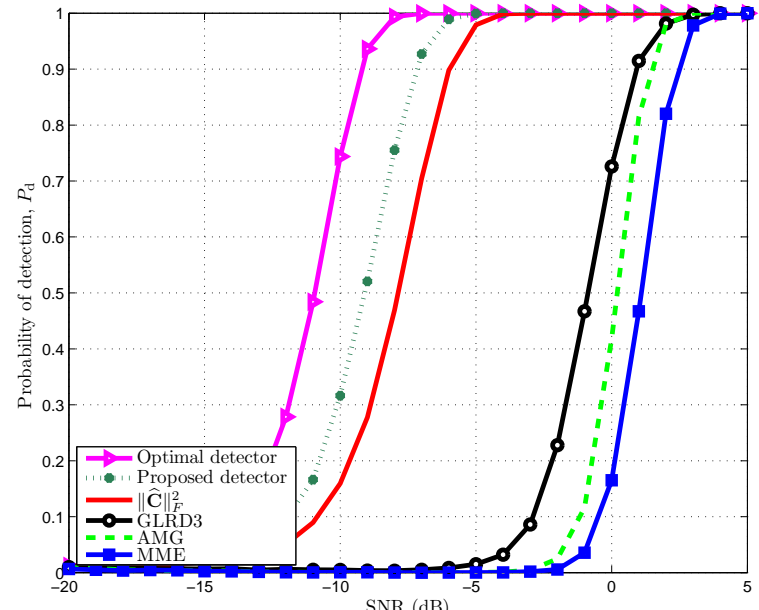

Fig. 9. Detection probability of different detectors versus SNR, for $P_{\mathrm{fa}}=$ $0.01, M=4, \Delta=\pi / 8$ and $L=100$.

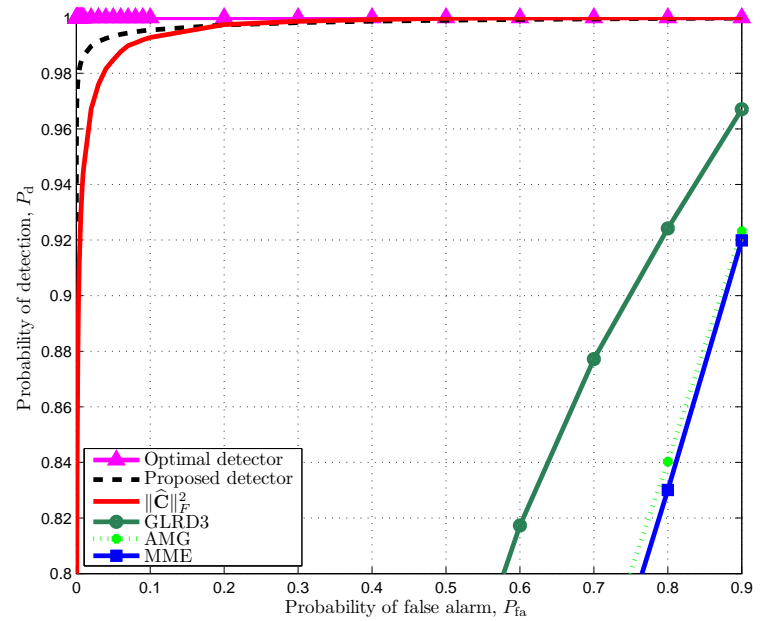

Fig. 10. The ROC curves of all detectors when $M=5$ and for $S N R=$ $-5(d B), \Delta=\pi / 8$, and $L=50$.

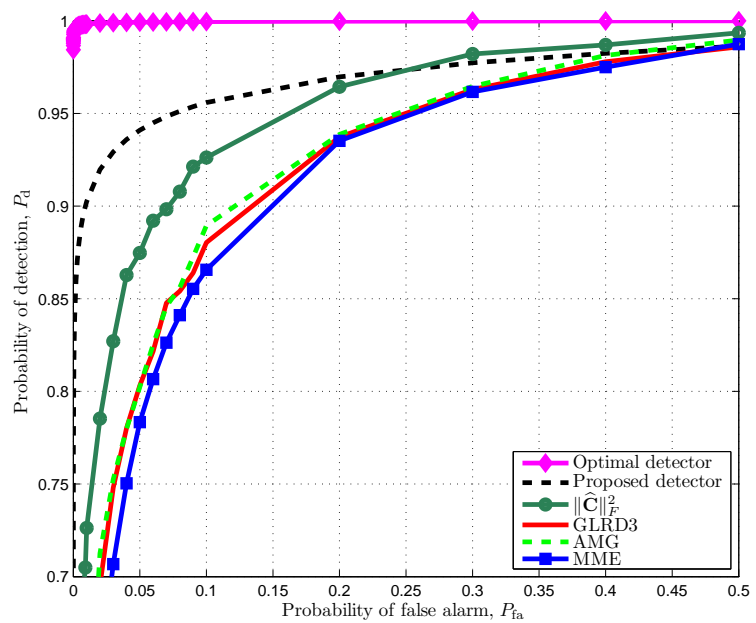

Fig. 11. The ROC curves of all detectors in case of calibration certainty, for $S N R=-6, M=4, L=100, \Delta=\pi / 8$ and $\boldsymbol{\Sigma}_{N}=\operatorname{diag}([1,1,1,1])$.

$S N R=-10(d B), \Delta=\pi / 8$ when received sensing samples 


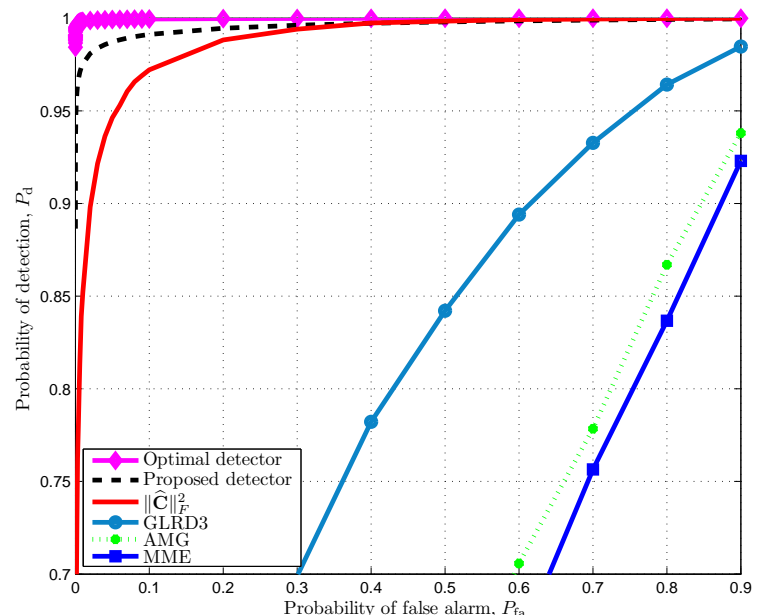

Fig. 12. The ROC curves of all detectors in uncalibration case when $S N R=$ -6 for optimal detector, $\|\widehat{\mathbf{C}}\|_{F}^{2}$ and our detector and $S N R=-3$ for others detectors, $M=4, L=100, \Delta=\pi / 8$ and $\boldsymbol{\Sigma}_{N}=\operatorname{diag}([1,0.75,0.5,0.25])$.

$L$ varies between three values. As observed, there is a good agreement between asymptotic analyze for performance and simulation-based results. In Fig. 3, we present comparison between analytical-based with simulation-based performance of proposed detector in special case when SU has two antennas (special case of Hadamard ratio detector) for some values of received samples, $L$ when $S N R=-6(d B)$ and $\Delta=\pi / 8$. We see that our analysis is completely exact in this case. For the general case, Fig. 4 and Fig. 5 respectively provide comparison between simulation and analytical CDF of the proposed decision statistic under $\mathcal{H}_{0}$ and $\mathcal{H}_{1}$. We have assumed that $M=4$, for $S N R=-5(d B), \Delta=\pi / 8$ and $\mathbf{w}=[1.6394,2.0275,2.0734,2.1830,1.2226,2.0924]^{T}$ so that $\mathrm{w}$ has been chosen randomly. As seen, our approximation becomes more accurate by increasing number of received samples $L$. In addition, Fig. 6 compares ROC curves based on simulation and asymptotic analytical results for $\|\widehat{\mathbf{C}}\|_{F}^{2}$. In this figure, we have considered that $M=4, S N R=-10(d B)$ and $\Delta=\pi / 8$. As can be observed, asymptotic results are accurate enough to use for solving of optimization problem . The available gap between curves is due to the asymptotic approximations used for deriving analytical results. This gap will be decreased by increasing the number of the samples at each antenna.

\section{B. Analysis of Effects of Received Sample Size, Number of Antennas and Beamwidth}

Here, we provide some simulation results to investigate the effects of receiving antennas number $M$, sample size of each antenna $L$ as well as maximum receiving beamwidth $\Delta$. Fig. 7 depicts the detection probability, $P_{\mathrm{d}}$ versus SNR for a varying values of $L$ when $M=3$ and $M=5$. In this figure false alarm probability, $P_{\mathrm{fa}}$, and maximum beamwidth have been set as 0.01 and $\Delta=\pi / 8$, respectively. As expected, the performance of the proposed detector improves by increasing the number of antennas or receiving samples. For example, the performance improvement at $P_{\mathrm{d}}=0.9$ and $L=200$ from $M=3$ to $M=5$ is about $5 \mathrm{~dB}$. In addition, at $P_{\mathrm{d}}=0.9$, the performance of proposed detector from $L=100$ to $L=250$ is improved about $4 \mathrm{~dB}$ and $3 \mathrm{~dB}$ for $M=3$ and $M=5$, respectively. As realized, the simulation results indicate that increasing the number of antennas, i.e., $M$, compared to increasing the number of samples, i.e., $L$, has a more substantial effect on the performance improvement of the proposed detector. For instance, the proposed detector at $L=100$ and $M=5$ has better performance than $L=250$ and $M=3$. Unfortunately, we cannot increasing $M$ arbitrary due to practical limitations. Fig. 8 shows the probability of detection of proposed detector versus SNR for varying receiving beamwidth, $\Delta$, for the case of $M=3$ and $M=5$ when $P_{\mathrm{fa}}=0.01$ and $L=200$. We can see that decreasing of $\Delta$ yields to improvement of performance of proposed scheme. Since, decreasing of beamwith results in increasing of channel coefficients correlation.

\section{Comparison Between Proposed Detector with Optimal Detector and Other Blind Detectors}

Now, we present some simulation results to compare our proposed scheme with proposed detector and some reported blind spectrum sensing techniques used as a benchmark as well as optimal detector. We have chosen four well performing known detectors: 1) the Generalized Likelihood Ratio Detector-3 (GLRD3) of [1]; 2) the Arithmetic-to-Geometric Mean (AGM) of [28]; 3) the Maximum-to-Minimum Eigenvalue (MME) of [29]; and 4) the special case of proposed detector with equal gains, i.e. $\|\widehat{\mathbf{C}}\|_{F}^{2}$, proposed in [17].

The detection probability versus SNR for our detector, optimal detector and four other detectors have been plotted in Fig. 9. In this figure, we considered $P_{\mathrm{fa}}=0.01, \Delta=\pi / 8$, $M=4$ and $L=100$. As observed, proposed detector at $P_{\mathrm{d}}=0.9$ outperforms respectively the GLRD3, AMG and MME about $7 \mathrm{~dB}, 8 \mathrm{~dB}, 9 \mathrm{~dB}$. These considerable difference in performance of proposed detector with other detectors is due to assumption of correlation among antennas and calibration errors in our detector. Fig. 10 indicates the ROC curves of all detectors when $M=5, S N R=-5(d B), \Delta=\pi / 8$, and $L=50$. As mentioned, calibration error and correlated antennas are inescapable in practice. Thus for other detectors these practical properties yield degradation of performance. In order to clarify better this result, we have presented simulation results considered the effect of correlation channel coefficients and calibration uncertainty on these different detectors.

Finally, the ROC curves for six detectors have been depicted in Fig. 11 and Fig. 12 which are based on calibration case and uncalibration error case, respectively. In Fig. 11, we assumed that $S N R=-6, M=4,=100, \Delta=\pi / 8$ and $\boldsymbol{\Sigma}_{N}=\operatorname{diag}([1,1,1,1])$. As seen, in the case of calibration certainty, difference between our detector and other detectors is small. If antennas are calibrated, we disregard this information in our model and thus, we may loss some performance. As an interesting results, we observe that our detector outperforms the other detectors even in this case. However, calibration errors are practically unavoidable as mentioned. Fig. 12 indicates that calibration uncertainty has significantly undesirable effect on other detectors while our 
detector is robust to it. In this figure, it is assumed that $S N R=-6$ for optimal detector, our detector and $\|\widehat{\mathbf{C}}\|_{F}^{2}$ and $S N R=-3$ for others detectors. In addition, $M=4, L=100$,

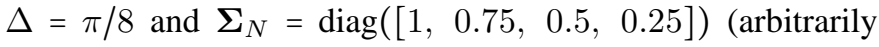
selected) have been considered.

\section{CONCLUSION}

In this paper, we studied the spectrum sensing problem for a CR multiantenna receiver which considers the correlation between the received channels at different antennas and uncalibrated antennas. As a benchmark, we first obtained the optimal NP detector and the asymptotical performance of optimal detector was provided. After that, we solved the sensing problem for practical scenarios in which SU has no knowledge about all of these parameters. In this case, we first considered the special case in which $\mathrm{SU}$ is equipped with only two antennas and derived sub-optimum detector based on GLRT which is a special case of Hadamard ratio test. Then, with the aid of derived results in special case, we proposed a general detector which combines weighted test statistics derived in special case among all two antennas. We evaluated the exact and asymptotic performance of detector with only two antennas in terms of detection and false alarm probabilities. Asymptotic expressions of false alarm and detection probabilities of proposed detector were obtained. In order to find optimum gains, we presented a convex optimization problem with closed-form solution. All analytical results have been verified by simulation results. The simulation results showed that proposed detector outperforms several recently-proposed detectors for CR using multiple antennas. In addition, we could demonstrate that our proposed detector has a better performance than the others even for the calibration case. Furthermore, it has been realized that our scheme will be more efficient when correlations among antennas are more stronger.

\section{APPENDICES}

\section{A. Performance of $T_{\mathrm{SPCL}}$}

We first find detection probability of special case where SU has two antennas, and then show that the false alarm probability is a special case of detection probability and derive it.

1) Detection Probability: In order to find distribution of decision statistic, we introduce some statistical results for the scatter matrix of the observations of $m^{t h}$ and $n^{t h}$ antennas, i.e. $\mathbf{R}(m, n)=\left(\begin{array}{ll}\mathbf{V}_{(m m)} & \mathbf{V}_{(m n)} \\ \mathbf{V}_{(n m)} & \mathbf{V}_{(n n)}\end{array}\right)$, under hypothesis $\mathcal{H}_{1}$. Then, we use them for calculation of $P_{\mathrm{d}}$. Under hypothesis $\mathcal{H}_{1}$, we have following Lemma.

Lemma 4. If $\mathbf{Y} \sim \mathcal{C N}\left(\mathbf{0}_{2 \cdot L}, \mathbf{I}_{L} \otimes \boldsymbol{\Lambda}(m, n)\right)$ where $\boldsymbol{\Lambda}(m, n) \triangleq$ $\left(\begin{array}{cc}\boldsymbol{\Sigma}_{1(m m)} & \boldsymbol{\Sigma}_{1(m n)} \\ \boldsymbol{\Sigma}_{1(n m)} & \boldsymbol{\Sigma}_{1(n n)}\end{array}\right)$, then:

1) $\mathbf{R}(m, n) \sim \mathcal{W}_{2}^{C}(L, \boldsymbol{\Lambda}(m, n))$ for $L>3$, where $\mathcal{W}_{2}^{C}(L, \boldsymbol{\Lambda}(m, n))$ is complex Wishart distribution with $L$ degrees of freedom and parameter matrix $\boldsymbol{\Lambda}$; and thus:

- $\mathbf{R}_{(22)}(m, n) \sim \frac{\boldsymbol{\Lambda}_{(22)}(m, n)}{2} \chi_{2 L}^{2}$ in which $\chi_{K}^{2}$ denotes Chi-square distribution with $K$ degrees of freedom.

$$
\begin{aligned}
- & \mathbf{R}_{(12)}(m, n) \quad \mid \quad \mathbf{R}_{(22)}(m, n) \\
& \mathcal{C N}\left(\mathbf{R}_{(22)}(m, n) \frac{\boldsymbol{\Lambda}_{(12)}(m, n)}{\boldsymbol{\Lambda}_{(22)}(m, n)}, \lambda_{s}^{2} \mathbf{R}_{(22)}(m, n)\right)
\end{aligned}
$$

in which $\lambda_{s}^{2} \triangleq \boldsymbol{\Lambda}_{(11)}(m, n)-\frac{\left|\boldsymbol{\Lambda}_{(12)}(m, n)\right|^{2}}{\boldsymbol{\Lambda}_{(22)}(m, n)}$ is the Schur complement of the $\boldsymbol{\Lambda}(m, n)$.

Proof: See [30, Ch. 8].

2) Let $r_{s}^{2} \triangleq \mathbf{R}_{(11)}(m, n)-\frac{\left|\mathbf{R}_{(12)}(m, n)\right|^{2}}{\mathbf{R}_{(22)}(m, n)}$ be the Schur complement of the $\mathbf{R}(m, n)$. Thus:

- $r_{s}^{2} \sim \frac{\lambda_{s}^{2}}{2} \chi_{2(L-1)}^{2}$.

- $r_{s}^{2} \Perp\left(\mathbf{R}_{(12)}(m, n), \mathbf{R}_{(22)}(m, n)\right)$.

Proof:

First of all note that $\mathbf{R}(m, n)=$ $\left(\begin{array}{ll}\mathbf{y}^{(m)^{H}} \mathbf{y}^{(m)} & \mathbf{y}^{(m)^{H}} \mathbf{y}^{(n)} \\ \mathbf{y}^{(n)^{H}} \mathbf{y}^{(m)} & \mathbf{y}^{(n)^{H}} \mathbf{y}^{(n)}\end{array}\right) . \quad$ Conditionally $\quad$ on $\mathbf{y}^{(n)}, \quad \mathbf{y}^{(m)} \sim \mathcal{C N}\left(\mathbf{y}_{n} \frac{\boldsymbol{\Lambda}_{(21)}(m, n)}{\boldsymbol{\Lambda}_{(22)}(m, n)}, \lambda_{s}^{2} \mathbf{I}_{L}\right) . \quad$ Let $\mathbf{P}_{\mathbf{y}^{(n)}} \triangleq \mathbf{y}^{(n)}\left(\mathbf{y}^{(n)^{H}} \mathbf{y}^{(n)}\right)^{-1} \mathbf{y}^{(n)^{H}}$ be the orthogonal projection on the column space of $\mathbf{y}^{(n)}$. Thus, $\mathbf{Q}_{\mathbf{y}^{(n)}}=\mathbf{I}_{L}-\mathbf{P}_{\mathbf{y}^{(n)}}$ is another orthogonal projection with the rank of $L-1$. Since $\mathbf{P}_{\mathbf{y}^{(n)}}^{H} \mathbf{P}_{\mathbf{y}^{(n)}}=\mathbf{P}_{\mathbf{y}^{(n)}}$ and $\mathbf{y}^{(n)}=\mathbf{P}_{\mathbf{y}^{(n)}} \mathbf{y}^{(n)}$, then we have

$$
\begin{aligned}
r_{s}^{2} & =\mathbf{y}^{(m)^{H}} \mathbf{y}^{(m)}-\mathbf{y}^{(m)^{H}} \mathbf{y}^{(n)}\left(\mathbf{y}^{(n)^{H}} \mathbf{y}^{(n)}\right)^{-1} \mathbf{y}^{(n)^{H}} \mathbf{y}^{(m)} \\
& =\mathbf{y}^{(m)^{H}}\left[\mathbf{I}_{L}-\mathbf{y}^{(n)}\left(\mathbf{y}^{(n)^{H}} \mathbf{y}^{(n)}\right)^{-1} \mathbf{y}^{(n)^{H}}\right] \mathbf{y}^{(m)} \\
& =\mathbf{y}^{(m)^{H}}\left[\mathbf{I}_{L}-\mathbf{P}_{\mathbf{y}^{(n)}}\right] \mathbf{y}^{(m)^{H}}=\mathbf{y}^{(m)^{H}} \mathbf{Q}_{\mathbf{y}^{(n)}} \mathbf{y}^{(m)} \\
& =\left[\mathbf{Q}_{\mathbf{y}^{(n)}} \mathbf{y}^{(m)}\right]^{H}\left[\mathbf{Q}_{\mathbf{y}^{(n)}} \mathbf{y}^{(m)}\right] .
\end{aligned}
$$

and

$$
\mathbf{R}_{(21)}(m, n)=\left[\mathbf{P}_{\mathbf{y}^{(n)}} \mathbf{y}^{(n)}\right]^{H}\left[\mathbf{P}_{\mathbf{y}^{(n)}} \mathbf{y}^{(m)}\right] .
$$

Let $\mathbf{Q}_{\mathbf{y}(n)}=\mathbf{U U}^{H}$ where $\mathbf{U}=\left(\mathbf{u}_{1}, \cdots, \mathbf{u}_{L-1}\right)$ is an orthogonal basis for its subspace and thus, $\mathbf{U}^{H} \mathbf{U}=\mathbf{I}_{L-1}$. Also, let $\mathbf{z} \sim \mathcal{C N}\left(0, \mathbf{I}_{L}\right)$. Clearly, we obtain from (24):

$$
r_{s}^{2}=\mathbf{y}_{m}^{H} \mathbf{U} \mathbf{U}^{H} \mathbf{y}^{(m)}=\lambda_{s} \mathbf{z}^{H} \mathbf{U} \mathbf{U}^{H} \mathbf{z} \lambda_{s}^{H}=\lambda_{s} w \lambda_{s}^{H} .
$$

where $w \sim \mathcal{W}_{1}^{C}(L-1)$. Thus, $r_{s}^{2} \sim \mathcal{W}_{1}^{C}\left(L-1, \lambda_{s}^{2}\right) \equiv$ $\frac{\lambda_{s}^{2}}{2} \chi_{2(L-1)}^{2}$, which does not depend on $\mathbf{y}^{(n)}$ and thus, on $\mathbf{R}_{(22)}(m, n)$. Please note that $\mathbf{Q}_{\mathbf{y}}{ }^{(n)} \mathbf{y}^{(n)}=\mathbf{0}$ and we can consider $\mathbf{y}^{(m)} \sim \mathcal{C N}\left(0, \lambda_{s}^{2} \mathbf{I}_{L}\right)$ in this proof. Now, in order to prove independency, we use $\mathbf{P}_{\mathbf{y}^{(n)}} \mathbf{y}^{(m)} \Perp$ $\mathbf{Q}_{\mathbf{y}^{(n)}} \mathbf{y}^{(m)}$ for given $\mathbf{y}^{(n)}$. Because, $\mathbf{P}_{\mathbf{y}}^{(n)} \mathbf{Q}_{\mathbf{y}}(n)=\mathbf{0}$ and

$$
\begin{aligned}
& \operatorname{var}\left[\left(\begin{array}{l}
\mathbf{P}_{\mathbf{y}^{(n)}} \\
\mathbf{Q}_{\mathbf{y}^{(n)}}
\end{array}\right) \mathbf{y}^{(m)} \mid \mathbf{y}^{(n)}\right] \\
& =\lambda_{s}^{2}\left[\left(\begin{array}{l}
\mathbf{P}_{\mathbf{y}^{(n)}} \\
\mathbf{Q}_{\mathbf{y}^{(n)}}
\end{array}\right)\left(\begin{array}{ll}
\mathbf{P}_{\mathbf{y}^{(n)}}^{H} & \left.\mathbf{Q}_{\mathbf{y}^{(n)}}^{H}\right)
\end{array}\right]\right. \\
& =\lambda_{s}^{2}\left(\begin{array}{cc}
\mathbf{P}_{\mathbf{y}^{(n)}} & \mathbf{0} \\
\mathbf{0} & \mathbf{Q}_{\mathbf{y}^{(n)}}
\end{array}\right) .
\end{aligned}
$$

Therefore, conditionally on $\mathbf{y}^{(n)}$, from (24) and (25) we conclude that $r_{s}^{2} \Perp \mathbf{R}_{(21)}(m, n)$. Hence, $r_{s}^{2} \Perp$ $\left(\mathbf{R}_{(21)}(m, n), \mathbf{R}_{(22)}(m, n)\right)$. 


$$
\begin{aligned}
& \mathbb{E}\left[f_{1}\left(r_{s}^{2}\right) \cdot f_{2}\left(\mathbf{R}_{(21)}(m, n), \mathbf{R}_{(22)}(m, n)\right)\right]=\mathbb{E}\left\{\mathbb{E}\left[f_{1}\left(r_{s}^{2}\right) \cdot f_{2}\left(\mathbf{R}_{(21)}(m, n), \mathbf{R}_{(22)}(m, n)\right) \mid \mathbf{y}^{(n)}\right]\right\} \\
& =\mathbb{E}\left\{\mathbb{E}\left[f_{1}\left(r_{s}^{2}\right) \mid \mathbf{y}^{(n)}\right] \mathbb{E}\left[f_{2}\left(\mathbf{R}_{(21)}(m, n), \mathbf{R}_{(22)}(m, n)\right) \mid \mathbf{y}^{(n)}\right]\right\}=\mathbb{E}\left\{\mathbb{E}\left[f_{1}\left(r_{s}^{2}\right)\right] \mathbb{E}\left[f_{2}\left(\mathbf{R}_{(21)}(m, n), \mathbf{R}_{(22)}(m, n)\right) \mid \mathbf{y}^{(n)}\right]\right\} \\
& =\mathbb{E}\left[f_{1}\left(r_{s}^{2}\right)\right] \mathbb{E}\left[f_{2}\left(\mathbf{R}_{(21)}(m, n), \mathbf{R}_{(22)}(m, n)\right)\right] .
\end{aligned}
$$

As can be easily seen, $r_{s}^{2}=\mathbf{R}_{(11)}(m, n)\left(1-\left|\hat{R}_{m, n}\right|^{2}\right)$ and so, we have

$$
\frac{\left|\hat{R}_{m, n}\right|^{2}}{1-\left|\hat{R}_{m, n}\right|^{2}}=\frac{1}{r_{s}^{2}} \frac{\left|\mathbf{R}_{(12)}(m, n)\right|^{2}}{\mathbf{R}_{(22)}(m, n)}
$$

According to Lemma 4, we can obtain

$$
\begin{array}{r}
\mathbf{R}_{(22)}(m, n)^{-\frac{1}{2}} \mathbf{R}_{(12)}(m, n) \mid \mathbf{R}_{(22)}(m, n) \\
\sim \mathcal{C N}\left(\mathbf{R}_{(22)}(m, n)^{\frac{1}{2}} \frac{\boldsymbol{\Lambda}_{(12)}(m, n)}{\boldsymbol{\Lambda}_{(22)}(m, n)}, \lambda_{s}^{2}\right)
\end{array}
$$

Hence, it can be proved that

$$
\frac{\left|\mathbf{R}_{(12)}(m, n)\right|^{2}}{\mathbf{R}_{(22)}(m, n)} \mid \mathbf{R}_{(22)}(m, n) \sim \frac{\lambda_{s}^{2}}{2} \chi_{2}^{2}(\delta)
$$

where $\delta \triangleq \frac{1}{\lambda_{s}^{2}} \frac{\left|\boldsymbol{\Lambda}_{(12)}(m, n)\right|^{2} \mathbf{R}_{(22)}(m, n)}{\boldsymbol{\Lambda}_{(22)}(m, n)^{2}}$ is the noncentrality parameter of noncentral Chi-square distribution. Now, using (29), (31) and Lemma 1, conditional on $\mathbf{R}_{(22)}(m, n)$, we will have [31, Ch. 12]:

$$
\frac{\left|\hat{R}_{m, n}\right|^{2}}{1-\left|\hat{R}_{m, n}\right|^{2}} \sim \frac{2}{2(L-1)} F(2,2(L-1) ; \delta)
$$

where $F\left(d_{1}, d_{2} ; \delta\right)$ is noncentral $\mathrm{F}$ distribution. Note that numerator and denominator of (29) are respectively a noncentral and a central Chi-square random variables which are statistically independent. We can write CDF of noncentral F distribution using $\mathrm{CDF}$ of central $\mathrm{F}$ distribution. With the aid of this property and by use of (32), we can write

$$
\begin{gathered}
P\left[\frac{2(L-1)}{2} \frac{\left|\hat{R}_{m, n}\right|^{2}}{1-\left|\hat{R}_{m, n}\right|^{2}}<\tau \mid \mathbf{R}_{(22)}(m, n), \mathcal{H}_{1}\right] \\
=\sum_{k=0}^{\infty} \exp \left(-\frac{\bar{\delta}}{2}\right) \frac{\bar{\delta}^{k}}{2^{k} k !} I_{\frac{2(1+k) \tau}{2(1+k) \tau+2(L-1)}}(1+k, L-1)
\end{gathered}
$$

where $\bar{\delta}$ is the $\delta$ conditional on $\mathbf{R}_{(22)}(m, n)$. Also, $I_{x}(a, b)=$ $\frac{1}{B(a, b)} \int_{0}^{x} t^{a-1}(1-t)^{b-1} \mathrm{~d} t$ is the regularized incomplete beta function. In order to obtain the unconditional distribution, we should take expectations on both sides of (33) with respect to the distribution of $\mathbf{R}_{(22)}(m, n)$. First, we require the distribution of $\delta$. Unconditionally, with the help of Lemma 1 , distribution of the noncentrality parameter can be simply derived as

$$
\delta \sim \frac{1}{2 \lambda_{s}^{2}} \frac{\left|\boldsymbol{\Lambda}_{(12)}(m, n)\right|^{2}}{\boldsymbol{\Lambda}_{(22)}(m, n)} \chi_{2 L}^{2}
$$

Let $\left|R_{m, n}\right|^{2}=\frac{\left|\boldsymbol{\Lambda}_{(12)}(m, n)\right|^{2}}{\boldsymbol{\Lambda}_{(11)}(m, n) \boldsymbol{\Lambda}_{(22)}(m, n)}$ be the actual correlation between $m^{t h}$ and $n^{t h}$ antennas. Therefore, (34) can be rewritten as

$$
\delta \sim \frac{1}{2} \frac{\left|R_{m, n}\right|^{2}}{1-\left|R_{m, n}\right|^{2}} \chi_{2 L}^{2}=\Gamma\left(L, \frac{\left|R_{m, n}\right|^{2}}{1-\left|R_{m, n}\right|^{2}}\right)
$$

If given $\delta, X$ is $\operatorname{Poisson}(\delta)$ and $\delta \sim \Gamma(p, \theta)$ in which $\Gamma(k, \theta)$ is Gamma distributed distribution with shape $k$ and scale $\theta$, then the marginal of $X$ is negative binomial, $X \sim n b(p,(1+$ $\theta)^{-1}$ ) [32]. Hence:

$$
\delta \sim \frac{1}{2} \frac{\left|R_{m, n}\right|^{2}}{1-\left|R_{m, n}\right|^{2}} \chi_{2 L}^{2}=\Gamma\left(L, \frac{\left|R_{m, n}\right|^{2}}{1-\left|R_{m, n}\right|^{2}}\right)
$$

It is clear that

$$
p_{k}=P(X=k)=\mathbb{E}[P(X=k \mid \delta)]=\mathbb{E}\left[\exp \left(-\frac{\delta}{2}\right) \frac{\delta^{k}}{2^{k} k !}\right]
$$

It can be easily demonstrated that

$$
p_{k}=\frac{L^{(k)}}{k !}\left(1-\left|R_{m, n}\right|^{2}\right)^{L}\left|R_{m, n}\right|^{2 k}
$$

where $x^{(n)}=x(x+1)(x+2) \cdots(x+n-1)$ is the rising factorial of $x$. Immediately, the expectation of (33) is calculated as

$$
\begin{aligned}
& P\left[\frac{2(L-1)}{2} \frac{\left|\hat{R}_{m, n}\right|^{2}}{1-\left|\hat{R}_{m, n}\right|^{2}}<\tau \mid \mathcal{H}_{1}\right] \\
= & \sum_{k=0}^{\infty} \mathbb{E}\left[\exp \left(-\frac{\delta}{2}\right) \frac{\delta^{k}}{2^{k} k !}\right] I_{\frac{2(1+k) \tau}{2(1+k) \tau+2(L-1)}}(1+k, L-1) \\
= & \sum_{k=0}^{\infty} p_{k} I_{\frac{2(1+k) \tau}{2(1+k) \tau+2(L-1)}}(1+k, L-1)
\end{aligned}
$$

If $\frac{d_{2} X}{d_{1}(1-X)} \sim F\left(d_{1}, d_{2}\right)$, then $X \sim \operatorname{Beta}\left(d_{1} / 2, d_{2} / 2\right)$ with the CDF of $I_{x}\left(d_{1} / 2, d_{2} / 2\right)$. Hence, (39) can be simplified as

$$
P\left[\left|\hat{R}_{m, n}\right|^{2}<\tau \mid \mathcal{H}_{1}\right]=\sum_{k=0}^{\infty} p_{k} I_{\tau}(1+k, L-1)
$$

So, we can obtain the detection probability as

$$
\begin{aligned}
P_{\mathrm{d}}^{\mathrm{SPCL}} & =P\left[\left|\hat{R}_{m, n}\right|^{2}>\tau \mid \mathcal{H}_{1}\right]=1-\sum_{k=0}^{\infty} p_{k} I_{\tau}(1+k, L-1) \\
& =\sum_{k=0}^{\infty} p_{k} I_{1-\tau}(L-1,1+k)
\end{aligned}
$$

2) False Alarm Probability: When samples of antennas are uncorrelated, i.e PU absence, the noncentrality parameter, $\delta$, is zero. Thus, (32) will be

$$
\frac{\left|\hat{R}_{m, n}\right|^{2}}{1-\left|\hat{R}_{m, n}\right|^{2}} \sim \frac{2}{2(L-1)} F(2,2(L-1))
$$

where $F\left(d_{1}, d_{2}\right)$ is central $\mathrm{F}$ distribution. We can easily demonstrate that the false alarm probability is

$$
\begin{aligned}
P_{\mathrm{fa}}^{\mathrm{SPCL}} & =P\left[\left|\hat{R}_{m, n}\right|^{2}>\tau \mid \mathcal{H}_{0}\right]=P\left[\left|\hat{R}_{m, n}\right|^{2}>\tau \mid \delta=0\right] \\
& =I_{1-\tau}(L-1,1)
\end{aligned}
$$

Thus, for a given $P_{\mathrm{fa}}^{\mathrm{SPCL}}$, the decision threshold is equal to $\tau=1-I^{-1}\left(L-1,1 ; P_{\mathrm{fa}}\right)$ in which $I^{-1}(a, b ; x)$ is the inverse function of the regulated incomplete beta function. According 
to the achieved results, we realize that decision threshold is a function of number of samples in each antenna and is independent of noise variances.

\section{B. Performance of Proposed Detector}

So far, we have investigated the exact performance of the detector in especial case when SU has two antennas. Now, we aim to find its performance for arbitrary number of antennas. To the best of our knowledge, there is no solution to find the exact performance of the proposed detector for the general case. But we can approximate the distribution of decision statistic by using an asymptotic approach. The accuracy of this approximation has been verified in Section V.

1) Detection Probability: Under $\mathcal{H}_{1}$, we approximate the distribution of decision statistic by a Gaussian distribution. A Gaussian distribution is completely described by its mean and variance. Thus, we need to find the mean and variance of $T_{\text {Prop }}$ under $\mathcal{H}_{1}$. To this end, we should first find the mean and variance of $\left|\hat{R}_{m, n}\right|^{2}$.

Lemma 5. The mean and variance of $\left|\hat{R}_{m, n}\right|^{2}$ under hypothesis $\mathcal{H}_{1}$, are respectively denoted as $\mu_{1}(m, n)$ and $\sigma_{1}^{2}(m, n)$, can be calculated as

$\mu_{1}(m, n)=1-\frac{L-1}{L}\left(1-\left|R_{m, n}\right|^{2}\right)_{2} F_{1}\left(1,1 ; L+1 ;\left|R_{m, n}\right|^{2}\right)$.

or asymptotically as

$$
\begin{aligned}
\mu_{1}(m, n)= & -\frac{L-1}{L(L+1)}\left|R_{m, n}\right|^{2}\left(1-\left|R_{m, n}\right|^{2}\right) \\
& +\left|R_{m, n}\right|^{2}+\frac{1-\left|R_{m, n}\right|^{2}}{L}+\mathcal{O}\left(L^{-2}\right) \\
\sigma_{1}^{2}(m, n)= & (L-1)\left(1-\left|R_{m, n}\right|^{2}\right)^{2} \times \\
& {\left[\frac{1}{L+1}{ }_{2} F_{1}\left(2,2 ; L+2 ;\left|R_{m, n}\right|^{2}\right)\right.} \\
& \left.-\frac{L-1}{L^{2}}{ }_{2} F_{1}^{2}\left(1,1 ; L+1 ;\left|R_{m, n}\right|^{2}\right)\right] .
\end{aligned}
$$

and asymptotically as

$$
\sigma_{1}^{2}(m, n)=\frac{2}{L}\left|R_{m, n}\right|^{2}\left(1-\left|R_{m, n}\right|^{2}\right)^{2}+\mathcal{O}\left(L^{-2}\right)
$$

Proof:

From (41) we have

$P\left[\left|\hat{R}_{m, n}\right|^{2}<\tau \mid \mathcal{H}_{1}\right]=\sum_{k=0}^{\infty} p_{k} P\left[\operatorname{Beta}(1+k, L-1) \leq \tau \mid \mathcal{H}_{1}\right]$

Thus

$$
\begin{aligned}
& P\left[1-\left|\hat{R}_{m, n}\right|^{2}<\tau \mid \mathcal{H}_{1}\right] \\
& =\sum_{k=0}^{\infty} p_{k} P\left[\operatorname{Beta}(L-1,1+k) \leq \tau \mid \mathcal{H}_{1}\right]
\end{aligned}
$$

Since $\mathbb{E}\left[X^{n}\right]=\frac{\alpha^{(n)}}{(\alpha+\beta)^{(n)}}$ if $X \sim \operatorname{Beta}(\alpha, \beta)$, then

$$
\begin{aligned}
& \mathbb{E}\left[\left(1-\left|\hat{R}_{m, n}\right|^{2}\right)^{n} \mid \mathcal{H}_{1}\right] \\
& =\sum_{k=0}^{\infty} \frac{L^{(k)}}{k !}\left(1-\left|R_{m, n}\right|^{2}\right)^{L}\left|R_{m, n}\right|^{2 k} \frac{(L-1)^{(n)}}{(L+k)^{(n)}}
\end{aligned}
$$

Let ${ }_{2} F_{1}(a, b ; c ; z)=\sum_{n=0}^{\infty} \frac{a^{(n)} b^{(n)}}{c_{(m)}^{(n)}} \frac{z^{n}}{n !}$ be hypergeometric function. Since $\frac{1}{(a+m)^{(n)}}=\frac{a^{(m)}}{a^{(n)}(a+n)^{(m)}}$, (50) can be rewritten as

$$
\begin{aligned}
\mathbb{E} & {\left[\left(1-\left|\hat{R}_{m, n}\right|^{2}\right)^{n} \mid \mathcal{H}_{1}\right] } \\
= & \sum_{k=0}^{\infty} \frac{L^{(k)}}{k !}\left(1-\left|R_{m, n}\right|^{2}\right)^{L}\left|R_{m, n}\right|^{2 k}(L-1)^{(n)} \frac{L^{(k)}}{L^{(n)}(L+n)^{(k)}} \\
= & \frac{(L-1)^{(n)}}{L^{(n)}}\left(1-\left|R_{m, n}\right|^{2}\right)^{L} \sum_{k=0}^{\infty} \frac{L^{(k)} L^{(k)}}{(L+n)^{(k)} k !}\left|R_{m, n}\right|^{2 k} \\
= & \frac{(L-1)^{(n)}}{L^{(n)}}\left(1-\left|R_{m, n}\right|^{2}\right)^{L}{ }_{2} F_{1}\left(L, L ; L+n ;\left|R_{m, n}\right|^{2}\right) \\
= & \frac{(L-1)^{(n)}}{L^{(n)}}\left(1-\left|R_{m, n}\right|^{2}\right)^{L}\left(1-\left|R_{m, n}\right|^{2}\right)^{n-L} \times \\
& { }_{2} F_{1}\left(n, n ; L+n ;\left|R_{m, n}\right|^{2}\right) \\
= & \frac{(L-1)^{(n)}}{L^{(n)}}\left(1-\left|R_{m, n}\right|^{2}\right)^{n}{ }_{2} F_{1}\left(n, n ; L+n ;\left|R_{m, n}\right|^{2}\right)
\end{aligned}
$$

Note that ${ }_{2} F_{1}(a, b ; c ; z)=(1-z)^{c-a-b}{ }_{2} F_{1}(c-a, c-b ; c ; z)$. Therefore, we can easily obtain the mean and variance of $\left|\hat{R}_{m, n}\right|^{2}$.

Since the $\left|\hat{R}_{m, n}\right|^{2}$ and $\left|\hat{R}_{k, \ell}\right|^{2}$ for $m \neq n$ and $k \neq \ell$ are correlated under hypothesis $\mathcal{H}_{1}$, we should find the covariance between antennas in order to calculate the variance. We can

(44)ind asymptotically desired correlations. To this end, we obtain asymptotic covariance matrix of $\left[\left|\hat{R}_{m, n}\right|^{2},\left|\hat{R}_{k, \ell}\right|^{2}\right]^{T}$ denoted as $\Psi$ using the Central Limit Theorem (CLT). Under hypothesis $\mathcal{H}_{1}$, the sample covariance matrix, $\mathbf{S}$, is complex Wishart distributed with $L$ degrees of freedom and parameter matrix $\boldsymbol{\Sigma}_{1}$. The moments of complex Wishart are calculated in [23]. Without loss of generality, let $\boldsymbol{\Sigma}_{1}=\left(\rho_{i j}\right)$ and $\rho_{i i}=1$ for $i=1, \cdots, M$. If number of received samples is large enough, using CLT and [23], we have

$$
\sqrt{L}\left[\left(\begin{array}{c}
\left|\mathbf{S}_{(m n)}\right|^{2} \\
\mathbf{S}_{(m m)} \\
\mathbf{S}_{(n n)} \\
\left|\mathbf{S}_{(k \ell)}\right|^{2} \\
\mathbf{S}_{(k k)} \\
\mathbf{S}_{(\ell \ell)}
\end{array}\right)-\left(\begin{array}{c}
\left|\rho_{m n}\right|^{2}+1 / L \\
1 \\
1 \\
\left|\rho_{k \ell}\right|^{2}+1 / L \\
1 \\
1
\end{array}\right) \mid \mathcal{H}_{1}\right] \rightarrow N(\mathbf{0}, \mathbf{\Omega})
$$

where $\Omega$ is a symmetric covariance matrix which its elements are given in (53). Define $\boldsymbol{\zeta} \triangleq\left[x_{1}, x_{2}, x_{3}, x_{4}, x_{5}, x_{6}\right]^{T}$ and $g(\zeta)=\left(\frac{\frac{x_{1}}{x_{2} x_{3}}}{\frac{x_{4}}{x_{5} x_{6}}}\right)$. Hence, we derive

$\nabla \mathbf{g}(\boldsymbol{\zeta})=\left(\begin{array}{cccccc}\frac{1}{x_{2} x_{3}} & -\frac{x_{1}}{x_{2}^{2} x_{3}} & -\frac{x_{1}}{x_{2} x_{3}^{2}} & 0 & 0 & 0 \\ 0 & 0 & 0 & \frac{1}{x_{2} x_{3}} & -\frac{x_{4}}{x_{5}^{2} x_{6}} & -\frac{x_{4}}{x_{5} x_{6}^{2}}\end{array}\right)$

Now, we apply Delta method and change (52) as

$$
\sqrt{L}\left[\left(\begin{array}{c}
\left|\hat{R}_{m, n}\right|^{2} \\
\left|\hat{R}_{k, \ell}\right|^{2}
\end{array}\right)-\left(\begin{array}{c}
\left|\rho_{m n}\right|^{2} \\
\left|\rho_{k \ell}\right|^{2}
\end{array}\right) \mid \mathcal{H}_{1}\right] \rightarrow N(\mathbf{0}, \mathbf{\Psi})
$$

in which $\boldsymbol{\Psi}=\nabla \mathbf{g}(\boldsymbol{\zeta}) \boldsymbol{\Omega} \nabla \mathbf{g}(\boldsymbol{\zeta})^{T}$ and equals to (56). Now, the covariance between $\left|\hat{R}_{m, n}\right|^{2}$ and $\left|\hat{R}_{k, \ell}\right|^{2}$ can be easily derived 


$$
\begin{gathered}
\boldsymbol{\Omega}_{(11)}=2\left|\rho_{m n}\right|^{2}+2\left|\rho_{m n}\right|^{4}+\frac{1}{L}\left(1+8\left|\rho_{m n}\right|^{2}+\left|\rho_{m n}\right|^{4}\right) ; \boldsymbol{\Omega}_{(12)}=\boldsymbol{\Omega}_{(13)}=2\left|\rho_{m n}\right|^{2}+\frac{1}{L}\left(1+\left|\rho_{m n}\right|^{2}\right) \\
\boldsymbol{\Omega}_{(14)}=2 \Re\left\{\rho_{\ell k} \rho_{k m} \rho_{n \ell} \rho_{m n}+\rho_{\ell k} \rho_{k n} \rho_{m \ell} \rho_{n m}\right\}+\frac{1}{L}\left(\left|\rho_{m \ell}\right|^{2}\left|\rho_{n k}\right|^{2}+\left|\rho_{n \ell}\right|^{2}\left|\rho_{m k}\right|^{2}+\right. \\
\left.2 \mathfrak{R}\left\{\rho_{\ell n} \rho_{m \ell} \rho_{n m}+\rho_{m k} \rho_{k n} \rho_{n m}+\rho_{k \ell} \rho_{\ell n} \rho_{n k}+\rho_{\ell k} \rho_{m \ell} \rho_{k m}\right\}\right) \\
\boldsymbol{\Omega}_{(15)}=2 \mathfrak{R}\left\{\rho_{k n} \rho_{m k} \rho_{n m}\right\}+\frac{1}{L}\left(\left|\rho_{m k}\right|^{2}+\left|\rho_{n k}\right|^{2}\right) ; \boldsymbol{\Omega}_{(16)}=2 \Re\left\{\rho_{\ell n} \rho_{m \ell} \rho_{n m}\right\}+\frac{1}{L}\left(\left|\rho_{m \ell}\right|^{2}+\left|\rho_{n \ell}\right|^{2}\right) \\
\boldsymbol{\Omega}_{(23)}=\left|\rho_{m n}\right|^{2} ; \boldsymbol{\Omega}_{(24)}=2 \Re\left\{\rho_{m k} \rho_{\ell m} \rho_{k \ell}\right\}+\frac{1}{L}\left(\left|\rho_{\ell m}\right|^{2}+\left|\rho_{k m}\right|^{2}\right) ; \boldsymbol{\Omega}_{(25)}=\left|\rho_{m k}\right|^{2} ; \boldsymbol{\Omega}_{(26)}=\left|\rho_{m \ell}\right|^{2} \\
\boldsymbol{\Omega}_{(34)}=2 \Re\left\{\rho_{n k} \rho_{\ell n} \rho_{k \ell}\right\}+\frac{1}{L}\left(\left|\rho_{\ell n}\right|^{2}+\left|\rho_{k n}\right|^{2}\right) ; \boldsymbol{\Omega}_{(35)}=\left|\rho_{n k}\right|^{2} ; \boldsymbol{\Omega}_{(36)}=\left|\rho_{n \ell}\right|^{2} \\
\boldsymbol{\Omega}_{(44)}=2\left|\rho_{k \ell}\right|^{2}+2\left|\rho_{k \ell}\right|^{4}+\frac{1}{L}\left(1+8\left|\rho_{k \ell}\right|^{2}+\left|\rho_{k \ell}\right|^{4}\right) ; \boldsymbol{\Omega}_{(45)}=\boldsymbol{\Omega}_{(46)}=2\left|\rho_{k \ell}\right|^{2}+\frac{1}{L}\left(1+\left|\rho_{k \ell}\right|^{2}\right) \\
\boldsymbol{\Omega}_{(56)}=\left|\rho_{k \ell}\right|^{2} ; \boldsymbol{\Omega}_{(22)}=\boldsymbol{\Omega}_{(33)}=\boldsymbol{\Omega}_{(55)}=\boldsymbol{\Omega}_{(66)}=1
\end{gathered}
$$

$$
\begin{aligned}
& \boldsymbol{\Psi}= \\
& \left(\begin{array}{cc}
2\left|\rho_{m n}\right|^{2}-4\left|\rho_{m n}\right|^{4}+2\left|\rho_{m n}\right|^{6}+\frac{1}{L}\left(1+4\left|\rho_{m n}\right|^{2}+5\left|\rho_{m n}\right|^{4}\right) & \psi \\
\psi & 2\left|\rho_{k \ell}\right|^{2}-4\left|\rho_{k \ell}\right|^{4}+2\left|\rho_{k \ell}\right|^{6}+\frac{1}{L}\left(1+4\left|\rho_{k \ell}\right|^{2}+5\left|\rho_{k \ell}\right|^{4}\right)
\end{array}\right)
\end{aligned}
$$

where

$$
\begin{aligned}
\psi= & 2 \mathfrak{R}\left\{\rho_{\ell k} \rho_{k m} \rho_{n \ell} \rho_{m n}+\rho_{\ell k} \rho_{k n} \rho_{m \ell} \rho_{n m}\right\}-2\left|\rho_{m n}\right|^{2} \mathfrak{R}\left\{\rho_{m k} \rho_{\ell m} \rho_{k \ell}+\rho_{n k} \rho_{\ell n} \rho_{k \ell}\right\} \\
& -2\left|\rho_{k \ell}\right|^{2} \mathfrak{R}\left\{\rho_{k n} \rho_{m k} \rho_{n m}+\rho_{\ell n} \rho_{m \ell} \rho_{n m}\right\}+\left|\rho_{m n}\right|^{2}\left|\rho_{k \ell}\right|^{2}\left(\left|\rho_{m k}\right|^{2}+\left|\rho_{n k}\right|^{2}+\left|\rho_{m \ell}\right|^{2}+\left|\rho_{n \ell}\right|^{2}\right) \\
& +\frac{1}{L}\left(\left|\rho_{m \ell}\right|^{2}\left|\rho_{n k}\right|^{2}+\left|\rho_{n \ell}\right|^{2}\left|\rho_{m k}\right|^{2}+\left(\left|\rho_{m n}\right|^{2}+\left|\rho_{k \ell}\right|^{2}\right)\left(\left|\rho_{m k}\right|^{2}+\left|\rho_{n k}\right|^{2}+\left|\rho_{m \ell}\right|^{2}+\left|\rho_{n \ell}\right|^{2}\right)\right)
\end{aligned}
$$

under hypothesis $\mathcal{H}_{1}$ as

$$
\begin{aligned}
& \operatorname{Cov}\left(\left|\hat{R}_{m, n}\right|^{2},\left|\hat{R}_{k, \ell}\right|^{2} \mid \mathcal{H}_{1}\right)=\frac{1}{L}\left(\left|R_{k, \ell}\right|^{2}\left|R_{m, n}\right|^{2}\left|R_{m, k}\right|^{2}\right. \\
+ & \left|R_{m, n}\right|^{2}\left|R_{n, k}\right|^{2}\left|R_{k, \ell}\right|^{2}+\left|R_{k, \ell}\right|^{2}\left|R_{m, n}\right|^{2}\left|R_{m, \ell}\right|^{2} \\
+ & \left|R_{m, n}\right|^{2}\left|R_{n, \ell}\right|^{2}\left|R_{k, \ell}\right|^{2}+2 \Re\left\{R_{\ell, k} R_{k, m} R_{n, \ell} R_{m, n}\right. \\
+ & \left.R_{\ell, k} R_{k, n} R_{m, \ell} R_{n, m}\right\}-2\left|R_{m, n}\right|^{2} \Re\left\{R_{m, k} R_{\ell, m} R_{k, \ell}\right. \\
+ & \left.R_{n, k} R_{\ell, n} R_{k, \ell}\right\}-2\left|R_{k, \ell}\right|^{2} \mathfrak{R}\left\{R_{k, n} R_{m, k} R_{n, m}\right. \\
+ & \left.\left.R_{\ell, n} R_{m, \ell} R_{n, m}\right\}\right)-\frac{1}{L^{2}}\left(-\left|R_{m, \ell}\right|^{2}\left|R_{n, k}\right|^{2}-\left|R_{n, \ell}\right|^{2}\left|R_{m, k}\right|^{2}\right. \\
+ & \left|R_{m, n}\right|^{2}\left(\left|R_{\ell, m}\right|^{2}+\left|R_{k, m}\right|^{2}+\left|R_{\ell, n}\right|^{2}+\left|R_{n, k}\right|^{2}\right) \\
- & 2 \Re\left\{R_{\ell, n} R_{m, \ell} R_{n, m}+R_{m, k} R_{k, n} R_{n, m}+R_{k, \ell} R_{\ell, n} R_{n, k}\right. \\
+ & \left.R_{\ell, k} R_{m, \ell} R_{k, m}\right\}+\left|R_{k, \ell}\right|^{2}\left(\left|R_{m, k}\right|^{2}+\left|R_{n, k}\right|^{2}\right. \\
+ & \left.\left.\left|R_{m, \ell}\right|^{2}+\left|R_{n, \ell}\right|^{2}\right)\right)
\end{aligned}
$$

Thus, the detection probability for general detector will be equal to

$$
P_{\mathrm{d}}^{\mathrm{prop}}=Q\left(\frac{\tau-\mathbf{w}^{T} \boldsymbol{\mu}_{1}}{\sqrt{\mathbf{w}^{T} \boldsymbol{\Theta} \mathbf{w}}}\right) .
$$

where $\boldsymbol{\mu}_{1}=\left[\mu_{1}(1,2), \mu_{1}(1,2), \cdots, \mu_{1}(M-1, M)\right]^{T}$ and
$\Theta$ is covariance matrix of $\mathbf{r}$ that each element could be derive asymptotically as (58). As realized, the probability of detection is dependent on number of received samples, number of antennas, correlation between antennas and received SNR in each antenna so that increasing of SNR or correlations yields an increment of detection probability. This analytical result will be verified experimentally in $\mathrm{V}$.

2) False Alarm Probability: In this part, we invoke the CLT ${ }_{2}$ to approximate behavior of sample covariance matrix, $\mathrm{S}$, as a Gaussian random matrix. First, consider the special case that $\mathrm{SU}$ is equipped with two antennas. If number of received samples, $L$, is large enough so that $\mathcal{O}\left(L^{-1 / 2}\right) \longrightarrow 0$, using (24) and (25) results in

$$
\begin{array}{r}
\sqrt{L} \mathbf{S}_{(m n)} \sim C N(0,1) \\
\mathbf{S}_{(m m)} \longrightarrow 1 \text { and } \mathbf{S}_{(n n)} \longrightarrow 1
\end{array}
$$

Thus, it can be easily shown that

$$
|\hat{R}(m, n)|^{2}=\left|\mathbf{S}_{(m n)}\right|^{2} \sim \frac{1}{2 L} \chi_{2}^{2}
$$

Therefore, $T_{\text {Prop }}$ is asymptotically distributed as a weighted Chi-square random variable which yields to mathematically 
untractable computation of weights $\mathbf{w}$. Thus, we fit $T_{\text {Prop }}$ with a Gaussian random variable and easily obtain

$$
P_{\mathrm{fa}}^{\mathrm{prop}}=Q\left(\frac{\tau-\mathbf{w}^{T} \mathbf{1} / L}{\|\mathbf{w}\| / L}\right) .
$$

When $\mathbf{w}=\mathbf{1}$, the general decision statistic under $\mathcal{H}_{0}$ is sum of independent Gamma random variables, it is a Gamma random variable as

$$
\|\widehat{\mathbf{C}}\|_{F}^{2} \sim \Gamma\left(N, \frac{1}{L}\right)
$$

Therefore, the false alarm probability of $\|\widehat{\mathbf{C}}\|_{F}^{2}$ is obtained as

$$
P_{\mathrm{fa}}=F_{\Gamma}\left(\tau ; N, \frac{1}{L}\right)=\frac{\Gamma\left(\frac{M(M-1)}{2}, \tau L\right)}{\Gamma(N)}
$$

where $F_{\Gamma}(\tau ; k, \theta)$ is complementary CDF of Gamma random variable with shape $k$ and scale parameter $\theta$. So, for a given $P_{\mathrm{fa}}=\varepsilon$, the decision threshold is $\tau=F_{\Gamma}^{-1}\left(\varepsilon ; N, \frac{1}{L}\right)$ which in $F_{\Gamma}^{-1}(\tau ; k, \theta)$ is inverse of $F_{\Gamma}(\tau ; k, \theta)$. There has been no analytical performance for $\|\widehat{\mathbf{C}}\|_{F}^{2}$ in literature, but we have proved that it is asymptotically distributed as a Gaussian random variable and a Gamma random variable under $\mathcal{H}_{1}$ and $\mathcal{H}_{0}$, respectively.

\section{REFERENCES}

[1] A. Taherpour, M. Nasiri-Kenari, and S. Gazor, "Multiple antenna spectrum sensing in cognitive radios," Wireless Communications, IEEE Transactions on, vol. 9, no. 2, pp. 814-823, 2010.

[2] R. López-Valcarce, G. Vazquez-Vilar, and J. Sala, "Multiantenna spectrum sensing for cognitive radio: overcoming noise uncertainty," in Cognitive Information Processing (CIP), 2010 2nd International Workshop on. IEEE, 2010, pp. 310-315.

[3] S. Atapattu, C. Tellambura, and H. Jiang, "Energy detection based cooperative spectrum sensing in cognitive radio networks," Wireless Communications, IEEE Transactions on, vol. 10, no. 4, pp. 1232-1241, 2011.

[4] S. Kim, J. Lee, H. Wang, and D. Hong, "Sensing performance of energy detector with correlated multiple antennas," Signal Processing Letters, IEEE, vol. 16, no. 8, pp. 671-674, 2009.

[5] R. Tandra and A. Sahai, "Snr walls for signal detection," Selected Topics in Signal Processing, IEEE Journal of, vol. 2, no. 1, pp. 4-17, 2008.

[6] L. Shen, H. Wang, W. Zhang, and Z. Zhao, "Multiple antennas assisted blind spectrum sensing in cognitive radio channels," Communications Letters, IEEE, vol. 16, no. 1, pp. 92-94, 2012.

[7] H. C. Nguyen, E. De Carvalho, and R. Prasad, "Spectrum sensing for cognitive radio based on multiple antennas," in Vehicular Technology Conference (VTC Spring), 2012 IEEE 75th. IEEE, 2012, pp. 1-5.

[8] D. Ramírez, G. Vazquez-Vilar, R. López-Valcarce, J. Vía, and I. Santamaría, "Detection of rank-p signals in cognitive radio networks with uncalibrated multiple antennas," IEEE Transactions on Signal Processing, vol. 59, no. 8, pp. 3764-3774, 2011.

[9] E. Hanafi, P. A. Martin, P. J. Smith, and A. J. Coulson, "Extension of quickest spectrum sensing to multiple antennas and rayleigh channels," Communications Letters, IEEE, vol. 17, no. 4, pp. 625-628, 2013.

[10] J. K. Tugnait, "On multiple antenna spectrum sensing under noise variance uncertainty and flat fading," Signal Processing, IEEE Transactions on, vol. 60, no. 4, pp. 1823-1832, 2012.

[11] L. Wei, P. Dharmawansa, and O. Tirkkonen, "Multiple primary user spectrum sensing in the low snr regime," 2013.

[12] D.-S. Shiu, G. J. Foschini, M. J. Gans, and J. M. Kahn, "Fading correlation and its effect on the capacity of multielement antenna systems," Communications, IEEE Transactions on, vol. 48, no. 3, pp. 502-513, 2000.

[13] C. Stevenson, G. Chouinard, Z. Lei, W. Hu, S. Shellhammer, and W. Caldwell, "Ieee 802.22: The first cognitive radio wireless regional area network standard," Communications Magazine, IEEE, vol. 47, no. 1, pp. 130-138, 2009.
[14] A. N. Mody, M. J. Sherman, R. Martinez, R. Reddy, and T. Kiernan, "Survey of ieee standards supporting cognitive radio and dynamic spectrum access," in Military Communications Conference, 2008. MILCOM 2008. IEEE. IEEE, 2008, pp. 1-7.

[15] L. Luo, P. Zhang, G. Zhang, and J. Qin, "Spectrum sensing for cognitive radio networks with correlated multiple antennas," Electronics letters, vol. 47, no. 23, pp. 1297-1298, 2011.

[16] M. Orooji, R. Soosahabi, and M. Naraghi-Pour, "Blind spectrum sensing using antenna arrays and path correlation," Vehicular Technology, IEEE Transactions on, vol. 60, no. 8, pp. 3758-3767, 2011.

[17] A. Lwshem and A.-J. Van der Veen, "Multichannel detection of gaussian signals with uncalibrated receivers," Signal Processing Letters, IEEE, vol. 8 , no. 4 , pp. 120-122, 2001.

[18] S. Sedighi, A. Taherpour, and J. Sala, "Spectrum sensing using correlated receiving multiple antennas in cognitive radios," Wireless Communications, IEEE Transactions on, vol. 12, no. 11, pp. 5754-5766, 2012.

[19] H. L. Van Trees, "Optimum array processing (detection, estimation, and modulation theory, part iv)," Wiley-Interscience, Mar, no. 50, p. 100, 2002.

[20] J. Salz and J. Winters, "Effect of fading correlation on adaptive arrays in digital mobile radio," Vehicular Technology, IEEE Transactions on, vol. 43, no. 4, pp. 1049-1057, 1994.

[21] T. S. Rappaport et al., Wireless communications: principles and practice. Prentice Hall PTR New Jersey, 1996, vol. 2.

[22] M. Browne and A. Shapiro, "The asymptotic covariance matrix of sample correlation coefficients under general conditions,", Linear Algebra Applications, vol. 83, pp. 169-176, 1986.

[23] D. Maiwald and D. Kraus, "Calculation of moments of complex wishart and complex inverse wishart distributed matrices," IEE ProceedingsRadar, Sonar and Navigation, vol. 147, no. 4, pp. 162-168, 2000.

[24] Z. Quan, S. Cui, and A. H. Sayed, "Optimal linear cooperation for spectrum sensing in cognitive radio networks," Selected Topics in Signal Processing, IEEE Journal of, vol. 2, no. 1, pp. 28-40, 2008.

[25] S. Krusevac, P. Rapajic, and R. A. Kennedy, "Channel capacity of multiantenna communication systems with closely spaced antenna elements," in Personal, Indoor and Mobile Radio Communications, 2005. PIMRC 2005. IEEE 16th International Symposium on, vol. 4. IEEE, 2005, pp. 2366-2370.

[26] J. W. Wallace and M. A. Jensen, "Mutual coupling in mimo wireless systems: A rigorous network theory analysis," Wireless Communications, IEEE Transactions on, vol. 3, no. 4, pp. 1317-1325, 2004.

[27] C. P. Domizioli, B. L. Hughes, K. G. Gard, and G. Lazzi, "Noise correlation in compact diversity receivers," Communications, IEEE Transactions on, vol. 58, no. 5, pp. 1426-1436, 2010.

[28] R. Zhang, T. Lim, Y.-C. Liang, and Y. Zeng, "Multi-antenna based spectrum sensing for cognitive radios: A glrt approach," Communications, IEEE Transactions on, vol. 58, no. 1, pp. 84-88, 2010.

[29] Y. Zeng, Y.-C. Liang, A. T. Hoang, and R. Zhang, "A review on spectrum sensing for cognitive radio: challenges and solutions," EURASIP Journal on Advances in Signal Processing, vol. 2010, p. 2, 2010.

[30] K. B. Petersen and M. S. Pedersen, "The matrix cookbook," Technical University of Denmark, pp. 7-15, 2008.

[31] K. Krishnamoorthy, Handbook of statistical distributions with applications. CRC Press, 2010.

[32] N. L. Johnson, A. W. Kemp, and S. Kotz, Univariate discrete distributions. Wiley-Interscience, 2005, vol. 444.

ACKNOWLEDGMENTS: The work of A. Taherpour and T. Khattab is supported by a grant from Qatar National Research Fund (a member of Qatar Foundation) under grant NPRP 6-1326-2-532. The statements made therein are the sole responsibility of the authors. The work of J. Sala-Alvarez was supported by projects: CENIT CEN-20101019 (THOFU), TEC2010-21245-C02/TCM (DYNACS), TEC2013-47020-C2-2-R (COMPASS) (Ministerio de Economia y Competitividad, Spanish Government) and 2014-SGR-60 AGAUR (Catalan Government). 